\title{
Computer vision analysis of unconstrained urban ground-level images
}

\author{
Eric Keiji Tokuda \\ THESIS PRESENTED \\ TO THE \\ Institute of Mathematics And Statistics \\ OF THE \\ University of SAO PAUlO \\ IN PARTIAL FULFILLMENT OF THE REQUIREMENTS \\ FOR THE DEGREE OF \\ Doctor of Philosophy in Computer Science \\ Program: Computer Science \\ Advisor: Prof. Dr. Roberto Marcondes Cesar Junior
}

The author gratefully acknowledges the financial support from FAPESP, grants \#2014/24918-0, \#2015/22308-2 and \#2016/12077-6

São Paulo, August 2019 


\section{Computer vision analysis of unconstrained urban ground-level images}

This version of the thesis contains the corrections and changes suggested by the Judging Committee during the defense of the original version of the work, which happened on July 11th, 2019. A copy of the original version is available at the Institute of Mathematics and Statistics of the University of São Paulo.

Judging committee:

- Prof. Dr. Roberto Marcondes Cesar Junior (advisor) - USP

- Prof. Dr. Roberto Hirata Junior (USP)

- Prof. Dr. Hélio Pedrini (Unicamp)

- Prof. Dr. João Paulo Papa (UNESP)

- Prof. Dr. Anderson Rocha (Unicamp) 


\section{Resumo}

TOKUDA, E. K. Visão Computacional aplicada a imagens urbanas em nível de chão sem restrições. 2019. 78 f. Tese (Doutorado) - Instituto de Matemática e Estatística, Universidade de São Paulo, São Paulo, 2019.

Atualmente, as imagens são geradas em larga escala e de forma descentralizada. Essa modalidade de dados carrega informações valiosas, mas extrair essas informações nem sempre é trivial. Nesta tese, definimos e propomos soluções a desafios de visão computacional ao utilizar imagens no nível do solo. O primeiro desafio é a anotação de alto custo para avaliar métodos de detecção de objetos. No contexto da degradação da imagem imposta pelo clima, a segunda questão é a falta de análise que avalie o impacto dos métodos de chuvas nos algoritmos de deteç̧ão de objetos em imagens chuvosas. O terceiro desafio é a confiabilidade dos resultados da estimativa de densidade de uma rede real de sensores. O surgimento de dados da rede de sensores motiva o último problema, de estimar a degradação urbana na cidade usando imagens em nível de solo. Esses desafios definem o escopo desta tese. Para o primeiro problema, propusemos uma abordagem com menor custo de anotação para comparação de detectores de objetos e a aplicamos em uma abordagem de aprendizado semi-supervisionada usando imagens de vigilância. Para enfrentar o desafio dois, estabelecemos um protocolo e realizamos uma extensa referência de detecção de objetos quando precedidas por métodos de remoção de chuva. Encontramos fortes indicadores de que métodos atuais de remoção de chuva não podem melhorar de maneira robusta a precisão da posterior detecção de objetos quando aplicado dessa maneira direta. A terceira questão foi abordada através da criação de um modelo de detecção probabilística para estabelecer limites teóricos para os erros das distribuições detectadas. A abordagem foi validada usando simulação e aplicada para calcular o mapa de densidade de pedestres em Manhattan. Para atacar o último problema, coletamos sistematicamente imagens públicas de São Paulo e segmentamos as regiões afetadas, como indicadores da degradação urbana da região. O código fonte foi integralmente publicado.

Palavras-chave: Computação Urbana, Visão Computacional, Detecção de objetos, Processamento de imagens, Cidades Inteligentes, Aprendizagem semi-supervisionada, câmeras de monitoramento, redes de sensores 


\section{Abstract}

TOKUDA, E. K. Computer vision analysis of unconstrained urban ground-level images. 2019. 78 p. Thesis (Ph.D.) - Institute of Mathematics and Statistics, University of São Paulo, São Paulo, 2019.

Nowadays, images are generated on a large scale and in a decentralized way. Such modality of data carries valuable information but extracting this information is not always trivial. In this thesis, we tackle computer vision challenges when using ground-level images. The first challenge is the high-cost annotation for evaluating object detection methods. In the context of image degradation imposed by weather, the second issue is the lack of analysis that evaluates the impact of de-raining methods to the object detection algorithms on rainy images. The third challenge is the evaluation of the reliability of the density estimation results from a real sensor network. The emergence of sensor network data motivates the last problem, of estimating the urban degradation in the city using city images. These challenges define the scope of this thesis. For the first problem, we proposed an approach with cheap annotation cost for object detectors comparison and we applied it in a semisupervised learning approach using surveillance images. To address challenge two, we established a protocol and performed an extensive benchmark of object detection preceded by de-raining methods. We find strong indicators that no current de-raining method can robustly improve the posterior object detection accuracy when applied in this naive way. The third issue was tackled by creating a probabilistic sensing model to establish theoretical bounds for the errors of the sensed distributions. The approach has been validated using simulation and applied to compute the pedestrian density map in Manhattan. To attack the last problem, we systematically collected public images of São Paulo and segmented the regions affected by tagging, as an indicator of the urban degradation of the region. The source code is fully released.

Keywords: Computer vision, urban computing, machine learning, object detection, image processing, smart cities, semi-supervised learning, sensor network, graffiti 


\section{Contents}

List of Abbreviations vii

$\begin{array}{ll}\text { List of Figures } & \text { ix }\end{array}$

List of Tables $\quad$ xiii

1 Introduction $\quad 1$

1.1 Motivation . . . . . . . . . . . . . . . . . . . . . 1

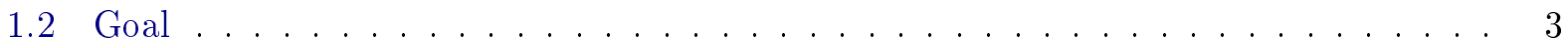

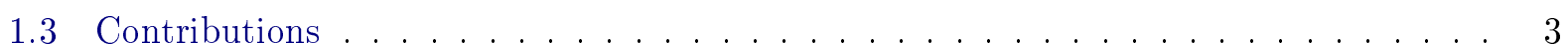

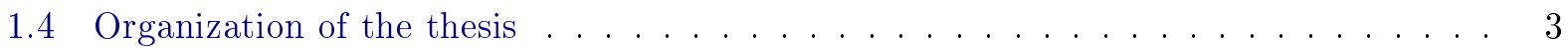

2 Concepts 5

2.1 Machine Learning . . . . . . . . . . . . . . . . . . 5

2.2 Object Detection . . . . . . . . . . . . . . . . . 6

3 Weak annotation for object detection learning and comparison $\quad 9$

3.1 Introduction . . . . . . . . . . . . . . . . . . . . 9

3.2 Material and Methods . . . . . . . . . . . . . . . . . . . . 10

3.2.1 Comparative Performance Assessment . . . . . . . . . . . . . . . . 11

3.3 Experimental Results and Validation . . . . . . . . . . . . . . . . . 11

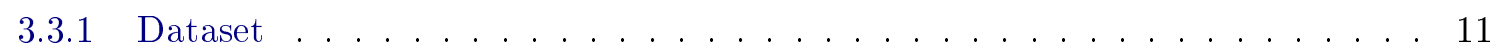

3.3.2 Validation 1: Uncontrolled Weather . . . . . . . . . . . . . . . . . 12

3.3.3 Validation 2: Rainy Days . . . . . . . . . . . . . . . . 12

3.4 Discussion and Concluding Remarks . . . . . . . . . . . . . . . . . . 13

4 Impact of de-raining to object detection $\quad \mathbf{1 5}$

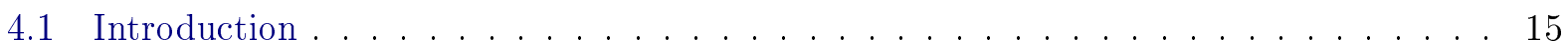

4.2 Proposed Evaluation Protocol . . . . . . . . . . . . . . . . . . . 17

4.3 Experimental Results . . . . . . . . . . . . . . . . . . . 17

4.4 Conclusion . . . . . . . . . . . . . . . . . . . . . . . . 19

5 Inaccurate Measurements in Sensor Networks $\quad 21$

5.1 Introduction . . . . . . . . . . . . . . . . . . . . 21

5.1 .1 Related Work . . . . . . . . . . . . . . . . . 22

5.2 Data and Proposed Method . . . . . . . . . . . . . . . . 23 
$5.2 .1 \quad$ Data . . . . . . . . . . . . . . . . . . . 23

5.2 .2 Person Detection . . . . . . . . . . . . . . . . . 25

5.2 .3 Sensing Model . . . . . . . . . . . . . . . . . . . 25

5.2 .4 Simulation . . . . . . . . . . . . . . . . . 27

5.3 Experimental Results . . . . . . . . . . . . . . . . . . . . . 28

5.3 .1 Person Detection Results . . . . . . . . . . . . . . . 28

5.3 .2 Simulation Calibration . . . . . . . . . . . . . . . . . 31

5.3 .3 Simulation Results . . . . . . . . . . . . . . . . . . . . . 33

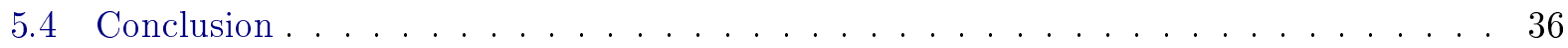

6 Graffiti Distribution from Ground-Level Images $\quad 37$

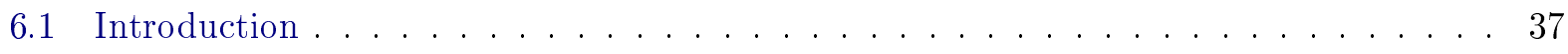

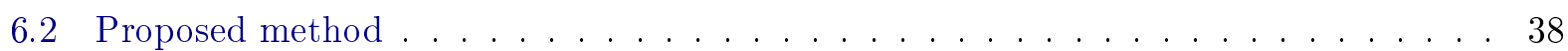

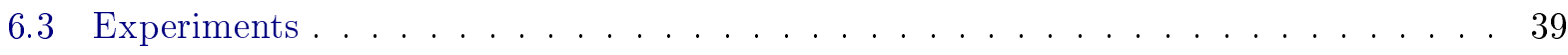

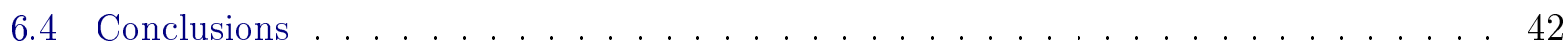

7 Conclusions $\quad 43$

$\begin{array}{ll}\text { A Proof of Metric Formula } & 47\end{array}$

A.1 Theoretical Asymptotic Error . . . . . . . . . . . . . . . . . . . 47

B Object Detection after Derain $\quad 51$

$\begin{array}{ll}\text { Bibliography } & 53\end{array}$ 


\title{
List of Abbreviations
}

\author{
CCTV Closed-Circuit Television \\ CDR Call Data record \\ CNN Convolutional Neural Networks \\ CV Computer Vision \\ DL Deep Learning \\ DPM Deformable Parts Model \\ FN False Negatives \\ FP False Positives \\ FPR False Positive Rate \\ GSV Google Street View \\ GT Ground Truth \\ GPU Graphics Processing Unit \\ HDI Human Development Index \\ ICT Information and Communications Technology \\ IoU Intersection over Union \\ ML Machine Learning \\ R-CNN Region-based Convolutional Neural Networks \\ SSD Single Shot MultiBox Detector \\ SSL Semi-supervised learning \\ SVM Support Vector Machines \\ TN True Negatives \\ TP True Positives \\ TPR True Positive Rate \\ WD Weak Detector \\ WSN Wireless Sensor Networks
}




\section{List of Figures}

1.1 Different views of the same location. From left to right, ground, aerial and satellite views of New York city. In this thesis, focus are given to ground-level images. Images

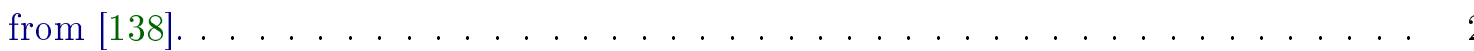

2.1 Main labelling possibilities for a dataset. These configurations are the focus of (a) unsupervised, (b) semi-supervised, (c) weakly-supervised and (d) supervised learning methods. . . . . . . . . . . . . . . . . . . . . . . .

2.2 Illustration of Table 2.1 for object detection. (a) corresponds to a correct detection, (b) to a incorrect detection, (c) a non-detection of an object and (d) to the correct non-detection. Even not perfectly enclosing the object, (a) is considered a match because the IoU is higher than the adopted detection threshold. . . . . . . . . . . .

3.1 Proposed framework. Frames are sampled and split into training and test sets. A weak detection is performed on the training set and used to train a new detector (SD). Quality control is performed both on the detections of the new detector on the test set as well as on the detections of the weak detector on the same set. . . . . . . 10

3.2 Sample of the collected dataset. The first row depicts images from the uncontrolled weather set while the second row shows images from the rainy set. . . . . . . . . . 12

3.3 Comparison of the cars detections performed by the $D P M, R C N N_{\text {all }}$ and $R C N N_{\text {rainy }}$, respectively, from left to right. The last frame indicates the pros (increased recall with two extra cars detected) and cons (a false positive leading to precision loss) of the proposed approach. . . . . . . . . . . . . . . . . . . 13

4.1 Visual degradation by adverse weather conditions. Images from [138] . . . . . . . . 15

4.2 Evaluation protocol. The surveillance images from rainy weather are processed using one of the five de-raining algorithms and is processed by one of the 4 object detection

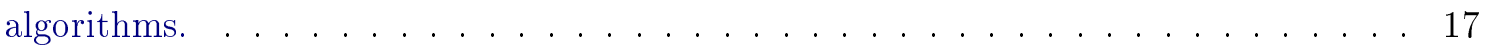

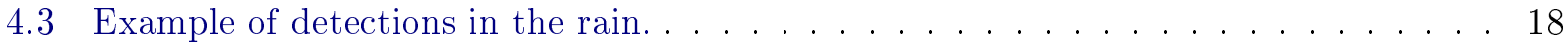

5.1 Temporal distribution of the collection of images by day of the week (left) and by hour of the day $($ right $) \ldots \ldots \ldots \ldots$. . . . . . . . . . . . . . 24

5.2 Geographical distribution of the number of pictures utilized in the region of Manhattan. The highest concentration of images are in the bottom and middle regions of map. 
5.3 Hypothetical person detection. The person on the center was not identified by the detector and is a false-negative. The rightmost detection is a false-positive. Underlying image provided by Carmera. . . . . . . . . . . . . . . . . 25

5.4 Left: An illustration of our simulation containing a sensor (center) moving through an environment with numerous person. Right: Each sensor moves with uniform speed $\nu_{s}$ and is able to sense people within a radius of $r$. Each sensing operation has a probability $p$ of correctly detecting each person and, on expectation, finds $\lambda$ false positives. People move with uniform speed $\nu_{p} \ldots \ldots \ldots \ldots \ldots \ldots$

5.5 Maps used in the simulation. Edges represent navigable paths. . . . . . . . . . . 28

5.6 Evaluation of R-FCN [25] for different ground-truth height thresholds. The utilized model has a Resnet-101 backbone [41] trained on the Pascal VOC 2007 dataset [30].

5.7 A comparison of the ground truth and the measured person count from the 600 tagged test images. While the actual true positive and false positive counts do not match the expected statistics (left), the total measured person count can be close to approximated as linear (right). It should be noted that this is only an approximation as, even taking sampling errors into account, the mean measured count do not fit a linear model. Error bars are the $95 \%$ confidence interval of the mean, calculated by assuming the sampling process described in Section 5.2.3. . . . . . . . . . . .

5.8 Visualization of the density of people in Manhattan. The scale of colors represent the relative density of people. The main figure depicts the heatmap over the island of Manhattan. The same heatmap is enlarged in the top-left of the figure to show the details of midtown and surrounding areas. Underlying map data taken from OpenStreetMap [88]. Not drawn to scale.

5.9 Person detection by the method in special cases. On the left, person detection in a crowded scene. On the right, pedestrians and non-pedestrians (a cyclist) are equally identified by the method.

5.10 Asymptotic error metric between the sensed and ground truth histograms in our simulation. Plotted as a function of the sensors true positive and expected number

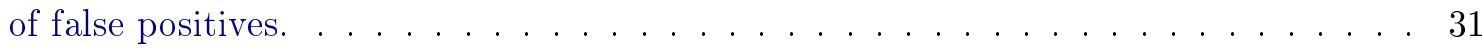

5.11 Interface created to visually explore the results of the simulation. . . . . . . . . . 33

5.12 Comparison of the asymptotic histograms error measured from the simulation experiments (blue dots) to the theoretical close form function and the approximate bound (please refer to Equation 5.9, red mesh) . . . . . . . . . . . . . .

6.1 Different types of graffiti. The wall pictured on the left contains a detailed and artistic drawing and it is reasonable to suppose that skill and time were necessary to paint it whereas the graffiti on the right is composed of words and symbols. In this work, there is special interest in the graffiti type on the right. Images from [38]. . . . . . . 37

6.2 Four views from the same geographical location. Images obtained from [38]. . . . . . 39

6.3 Training and validation loss of the [40]-based model during the training stage. The highlighted points (epoch 30) correspond to the final model training and validation accuracy. . . . . . . . . . . . . . . . . . . . .

6.4 Coverage of the utilized images using [38] images. Dots represents existence of pictures in the region. Low coverage can be observed in the bottom of the map. . . . . . 
6.5 Sample of the graffiti detections. The values depicted represent the confidence of the detections. . . . . . . . . . . . . . . . . . . 41

6.6 (Left) Human development Index from São Paulo districts in 2007 [117]. (Right) Relative graffiti level in São Paulo city. The divisions represent the city districts and the colors in logarithmic scale express the relative graffiti level, according to Equation 6.2. Bottommost regions were not considered given the unavailability of data in the region. . . . . . . . . . . . . . . . . . . 41

B.1 Sample of the detection on rainy images. . . . . . . . . . . . . . . 51 


\section{List of Tables}

2.1 Confusion matrix. Prediction matches (in blue) and errors (in red) according to the ground-truth values. . . . . . . . . . . . . . . . . 5

3.1 Quality control over the Validation 1 and Validation $2 \ldots \ldots \ldots \ldots$

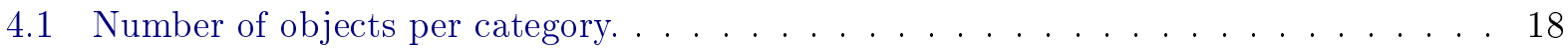

4.2 Average no-reference evaluation results after de-raining processing. . . . . . . . . 18

4.3 De-raining detection results per category. . . . . . . . . . . . . . . . . 19

5.1 Comparison of the numbers of images utilized to other popular datasets. . . . . . . 23

5.2 Parameters of the simulation along with the values we used in the experiments. . . . 34

6.1 Acquisition year of the geographical location analyzed. Metadata provided by [38]. 40 


\section{Chapter 1}

\section{Introduction}

\subsection{Motivation}

In the last decade, the urban population surpassed half of the world's population and projections estimate it to reach two-thirds of the global total population in a few decades [129]. This phenomenon is mainly propelled by the natural increase of the population and by the rural-urban migration. The migration to urban spaces has several factors including the search for better labor and education opportunities, improved public health, higher female labor participation, more social mobility and fertile land for art. Notwithstanding, managing such conglomerates is a daunting task. There are several challenges associated with the environment, including issues such as pollution and the deliberate use of natural resources. Today around $25 \%$ of the world population live in regions with lack of access to clean water, basic sanitation and in insufficient living area. In Sub-Saharian Africa, the ratio of people living in such conditions was close to $72 \%$ in 2003 [94]. Such hostile environments facilitate the outbreak of disease epidemics, civil disruptions and economic fluctuations. All these urban issues deserve proper local and national authorities' attention, as well as from the community.

At the same time the world becomes urban, it also becomes digital. The first feature of the data that may come to one's mind is the volume. In fact, an unprecedented magnitude of data already exists, at the scale of zettabytes. However, data comes also with velocity, given the increase of data throughput in many areas such as Particle Physics and Astronomy. Thirdly, data comes with heterogeneity given the different sources of information, abstraction and structuredness. Lastly, data veracity is questionable, given the common presence of noise and errors [18].

In this scenario, researchers started exploring novel data-centric ways to solve urban problems. For instance, the availability of data paved the way in social sciences. Until recently, humanconducted surveys, often expensive, unstructured or of coarse scale represented a major tool for the field [20]. Today, for instance data collected from a ride-sharing service can give a broad picture of the population everyday commute [32]. Such use, however, requires mining the raw data available. The knowledge extraction is a personalized and complex task, given the heterogeneity of the data available. Data must be systematically collected, filtered and analyzed [18]. In this context, the data science emerged as an important field and where ML plays an important role. Credit card transactions have long been used by banks to analyze spendings profiles and loan standards [89]. Today, it is a common practice to track consumers' data and feed them to ML algorithms for model refinement.

Visual data represent an important way to promote content given the efficient human's visual cognition. In special, pictures have the power to communicate the reality and feelings that could not for instance be expressed by other means. Thanks to advances in fields such as astronomy and microelectronics, pictures can be obtained in different scales and resolutions (see Figure 1.1). Researchers can view, even if indirectly, from sub-atoms particles to whole galaxies. As efficiently used in the study of natural disasters, satellite images provide a particular way to analyze a large region. Aerial images have comparatively smaller coverage and higher resolution and their acqui- 

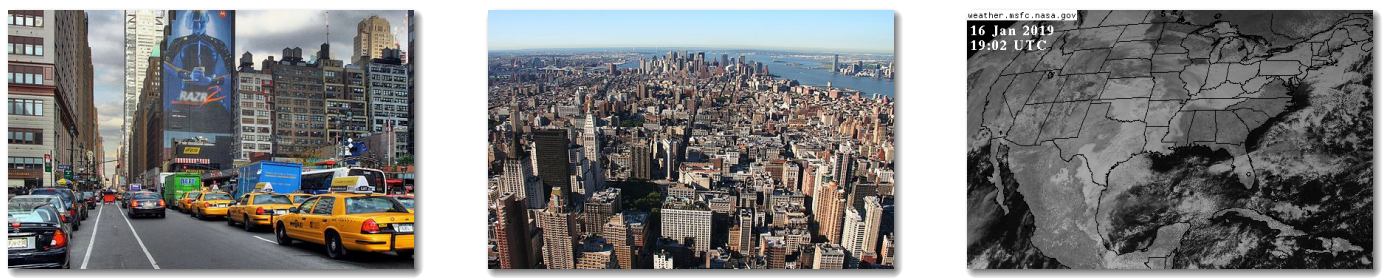

Figure 1.1: Different views of the same location. From left to right, ground, aerial and satellite views of New York city. In this thesis, focus are given to ground-level images. Images from [138].

sition apparatus are more accessible to end-users. This kind of image has been historically used in military and agricultural applications and gained special popularity with the rise of unmanned aerial vehicles. Ground-level images are by far the most popular modality of images, partly due to the popularity of camera-equipped telephones. Given the concentration of the population in cities, the majority of the ground-level images are generated in these conglomerates. Ground-level images are generated in systematic ways, such as by a sensors network or in decentralized ways, like pictures taken by social media users.

Such an increase in the urban visual data available coupled with the existence and emergence of urban problems may motivate the general question:

What are the challenges in using ground-level images to tackle urban issues?

Computer vision plays a key role in the knowledge extraction from images. Recent supervised learning methods report high accuracies in different tasks [116] but they require annotated training data, which for tasks such as object detection may be hard and time-consuming to obtain. In the particular case of the comparison of two object detection methods, one may ask Question 01) How to compare the performances of two object detection approaches with minimal annotation work?

The oldest systematic acquisition of images is through a network of static cameras. CCTV cameras have long been considered an important source of information. It is of great utility for the public safety authorities to have the city monitored and CCTV cameras represent an important tool in this task. Cities such as Beijing are gradually increasing their cameras network in the city [84]. When using CCTV cameras for knowledge extraction, there are known challenges associated with it. For instance, outdoor cameras are subject to weather variations. The most common weather adversity that affects visual appearance is the rain. So a question that may appear when handling this kind of imagery is: Question 02) Can the current single image de-raining methods help in the task of object detection in low-resolution images in rainy weather?

Despite recent computer vision methods achieve impressive performances in benchmarks, results are far behind in unconstrained scenarios. There are several challenges in unconstrained environments such as the already mentioned weather, illumination, and jittering of the camera. Networks of cameras obtain city images from outdoors, with high coverage and frequency which may affect the computer vision task. This faulty processing of the data may make one wonder about the reliability of the results obtained. The following question may arise: Question 03) Is the information obtained from a network of sensors that inaccurately counts the particles nearby reliable?

In a scenario of mobile cameras, like a network of vehicles equipped with high-resolution cameras travelling around the city, one may wonder Question 04) How to estimate the pedestrian distribution in a region using a set of unconstrained ground-level and irregularly sampled pictures of this region?

The most well known public service that provides such type of data is Google Maps. Countless applications are using these kinds of data. Few works, however, have utilized their work to study the vandalism. One may wonder: Question 05) Can ground-level images obtained from a wireless sensors network be used to compute the graffiti map of a city?

These questions motivate the goal of this work. 


\subsection{Goal}

The goal of this thesis is to present some novel methods developed to analyze and model urban ground-level images using computer vision.

\subsection{Contributions}

This project was geared towards the analysis of the challenges and applications of urban pictures to solve urban issues. The main contributions can be summarized as:

a) a novel approach for comparing existing object detectors and learn new ones that requires just the inspection of the detections;

b) an unprecedented benchmark of the impact of state-of-the-art de-raining algorithms to the object detection in low-resolution images;

c) a new model model of sensor networks that contemplates the sensing inaccuracies;

d) a practical way of computing the pedestrian distribution using ground-level images obtained from a wireless sensor network;

e) a new way of estimating urban vandalism through the use of images.

\subsection{Organization of the thesis}

The structure of this document is publication-oriented what means that each contribution above is described in a separate chapter of the thesis, except by $c$ ) and $d$ ) which are both covered in Chapter 5. Each chapter is an adapted version of a produced paper. Additionally, Chapter 2 presents fundamental concepts and Chapter 7 presents the finals remarks. 


\section{Chapter 2}

\section{Concepts}

In this chapter, fundamental concepts, definitions and notations related to the problems to follow are established and explained. Initially machine learning concepts are defined followed by a review of important object detection methods.

\subsection{Machine Learning}

Machine learning (ML) methods try to learn from data and are classically divided into three groups: reinforcement learning, supervised learning and unsupervised learning. Reinforcement learning assumes a learning agent that earns rewards according to its interaction with the environment in which there is no guidance on which are the best set of actions. Supervised learning in turn has the support of existing annotated data to guide the whole learning process and finally, unsupervised learning, does not have this assistance [116]. The task of annotating the data may be laborious and many crowdsourcing services emerged as a way to provide such functionalities [3]. These services though are not always applicable because they may require experts in the field to perform the task and also there may be human bias involved. A common scenario is a large volume of data with just part of it annotated. Initial works in this scenario considered the disposal of the annotations reducing the problem to an unsupervised learning one or discarding the non-annotated data reducing it to a classic supervised learning problem [17]. More recently, the so-called semi-supervised learning (SSL) methods started to make structural assumptions about the data to leverage the unlabeled instances. In a different setting, instead of precise annotations, only a less precise or partial annotation (weak label) of each instance is provided. Weakly supervised learning methods [149] handle this kind or problem. See Figure 2.1 for an illustration of these possibilities.

A common way to assess ML experiments results is through the use of precision and recall. Assuming a simple ML prediction task in which the outcome is either positive or negative and the existence of a ground-truth (GT), TP refers to the number of predicted positives that were correctly predicted and FP to the incorrectly predicted. Similarly TN and FN refer to the number of correctly and incorrectly, respectively, predicted negatives (see Table 2.1). These measures can be used in raw counts or may be expressed in the relative terms of precision, recall and $\mathrm{F}$ measure (Equation 2.1). Precision measures how reliable are the predictions with respect to all predictions and recall measures how reliable they are with respect to all the positive cases.

Table 2.1: Confusion matrix. Prediction matches (in blue) and errors (in red) according to the ground-truth values.

\begin{tabular}{lcc}
\hline & GT positive & GT Negative \\
\cline { 2 - 3 } Predicted positive & true positive & false positive \\
Predicted negative & false negative & true negative \\
\hline
\end{tabular}




$$
\text { precision }=\frac{T P}{T P+F P} \quad \text { recall }=\frac{T P}{T P+F N} \quad F 1=2 \frac{\text { precision } \times \text { recall }}{\text { precision }+ \text { recall }}
$$

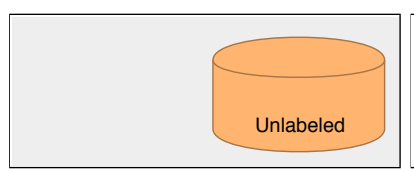

(a) No labels.

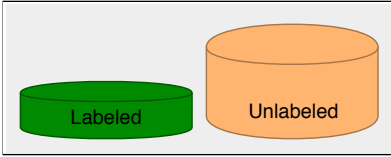

(b) Some labels.

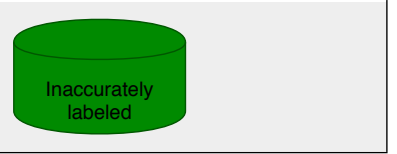

(c) Incomplete labels.

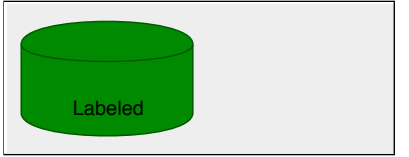

(d) (Full) labels.

Figure 2.1: Main labelling possibilities for a dataset. These configurations are the focus of (a) unsupervised, (b) semi-supervised, (c) weakly-supervised and (d) supervised learning methods.

$\mathrm{CV}$ has been marked by the proposal of hand-engineered features, such as SIFT [75] and HoG [26] used in conjunction with ML algorithms like SVM [14] and these fields were largely independent [116]. The vision task performance thus was very dependent on a complex feature handcrafting (feature engineering) process. Today, however, CV researchers can propose methods with state-of-the-art results with no need of designing features anymore. That is mainly due to a paradigm shift brought by deep learning (DL). A simplistic definition of a DL method is of a neural network with multiple hidden layers [59] and its success is commonly attributed to the availability of data and the processing in graphics processing units (GPU).

Convolutional neural networks (CNN) are the most popular type of DL methods. CNN are mainly composed of three types of layers: convolutional layer, that are equivalent to filters that convolve over the previous layer and generates an activation map; non-linearity layer, that adds a non-linear operation; and pooling layer, responsible for reducing the number of parameters. Fully connected layer is a convolutional layer with kernel size equal to the input size. The loss measures the difference between the expected and the obtained results of a forward-pass and is defined by a loss function [59].

\subsection{Object Detection}

Object detection is a CV task defined as the problem of locating the instances of an object of interest in an image [116]. It remains one of the most challenging tasks in computer vision due to problems such as occlusion, intraclass variance and pose variation. Each detection is fully described by the category, score and the bounding box $(B)$. The bounding box is described by a rectangle with proper shape and localization in the image. The number of the detected bounding boxes that matches the ground truth is a fundamental assessment criterion. A perfect match occurs when the detected bounding box intersects $100 \%$ with the ground-truth. A metric commonly adopted for object detection is the Intersection over Union (IoU) or Jaccard Index, defined as:

$$
I o U=\frac{\left|B_{\text {det }} \cap B_{G T}\right|}{\left|B_{\text {det }} \cup B_{G T}\right|}
$$

Assuming the detection as positive and the background as negative, and given a hit criterion, the performance of a detector in an image can be described by the numbers of correctly identified objects (TP), incorrectly identified objects (FP), backgrounds incorrectly identified as objects (FN), and by the backgrounds correctly identified (TN) in the image. The TN in object detection is generally not calculable, given the substantial number of possibilities for the background.

The Deformable Parts Model (DPM) [31] is considered one of the most influential paper in computer vision prior to DL. It proposes the recognition of an object by finding its constituent parts and analyzing the relative disposition of them. Object templates (models) are created based on multi-scale HoG features. Each template consists of a coarse-scale HoG feature of the object, higher-scale HoG features representing the part filters and a displacement penalty map for each 


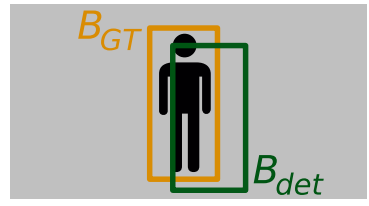

(a) True positive

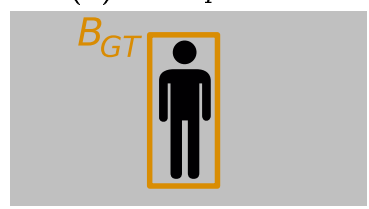

(c) False negative

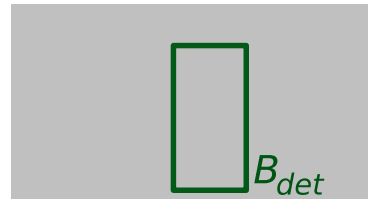

(b) False positive

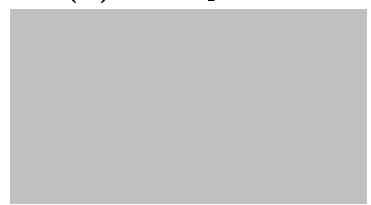

(d) True negative

Figure 2.2: Illustration of Table 2.1 for object detection. (a) corresponds to a correct detection, (b) to a incorrect detection, (c) a non-detection of an object and (d) to the correct non-detection. Even not perfectly enclosing the object, (a) is considered a match because the IoU is higher than the adopted detection threshold.

part. The score of a candidate is thus given by the sum of the filters responses penalized by the divergence between the target's and the template parts disposition.

The first DL results of great impact appeared in CV classification challenges [55]. At the time, the task of finding region proposals or also called region candidates was already a well studied problem in CV [56]. Shortly after, the first popular two-stage detection was proposed [100]. Twostage object detection are characterized by dividing the task of object detection into two tasks: object proposal and classification. That term is in contrast to methods like [98, 72, 69] that does the classification of a fixed number of boxes of pre-defined sizes.

The most famous method based on region proposal [100] is a modified version of the former [37]. In the original method [37], the task of region proposals is relied on an external method such as [128] to find thousands of region candidates. Each region proposal is then resized to a fixed dimension, a CNN is used for feature extraction and finally an SVM is used for classification of the candidate. [100] achieved better and faster results in PASCAL VOC 2007 [30] compared to its predecessor. The main difference brought by Faster R-CNN (FRCNN) is the introduction of a unified architecture that shares region proposal convolutional layers with object detection networks.

RetinaNet [69] is a one-stage object detector which main feature is the incorporation of a modulating factor to the cross-entropy loss. The so-called focal loss was motivated by the learning drift of one-stage detectors caused by the generally high class imbalance of foreground-background ratio. Focal loss reduces the relative loss for well-classified examples and consequently put more focus on hard, misclassified examples. RetinaNet architecture is composed of a multi-scale subnetwork for feature extraction, two subnetworks for classification and bounding box regression. They reported state of the art results at the time on MS-COCO object detection test-dev benchmark [104].

The Single Shot multibox Detector (SSD) [72] is one the most popular recent one-stage object detector. It extracts multi-scale features maps using a convolutional neural network and apply convolution filter for the detection, to compute what they call location and class scores. The multiscale feature allows detection of objects of different sizes. A fundamental concept in FRCNN is of anchors and here as well the authors define default boxes of different aspect ratios. Additional strategies to improve learning include non maximal suppression, hard negative mining and data augmentation strategies. The original implementation has the same architecture as [55] as feature extractor. 


\section{Chapter 3}

\section{Weak annotation for object detection learning and comparison}

The main findings of this chapter have been published in [121], in which this Chapter is based on.

\subsection{Introduction}

The availability of labeled instances is a fundamental requirement for training supervised learning methods [48]. In applications such as object detection, annotated instances are hard and time consuming to obtain because in most cases experts are required to manually generate these data. An option is to use crowd-sourcing services such as [3, 23]. Empirical results including [104] show the effectiveness of such services but it can be costly and the quality may fall short depending on the kind of task $[112,107]$. Supervised learning methods differ from unsupervised ones by the use of labeled data in the training process $[48,83]$. Semi-supervised methods assume the availability of both kinds of data in the training set and try to effectively use them for better performance [17].

In the semi-supervised approach proposed by [102] a classifier incrementally auto-feeds its best results to adjust its parameters [102]. Despite the automatic fine-tuning, the evaluation of the results requires full manual annotation to serve as ground-truth. Our work differs from [102] by the requirement of weak annotation instead to evaluate the method generated. Another difference is the single-pass update instead of incremental updates. Another semi-supervised work [81] assumes weakly-labeled frames to find objects in videos. It uses tracking and temporal consistency to improve their results. Our approach does not require tracking, thus avoiding possible problems such as drifting.

Objects detection is a very active research area [116]. The Deformable Parts Model (DPM) is a notable object detection method proposed by [31]. It identifies an object through its constituent parts and the corresponding spatial dispositions. The seminal work of Regions with Convolutional Neural Networks (RCNN) [100] proposes a pipeline of finding class-agnostic regions of interest and then classifying them using state-of-art classifiers. The fine-tuning of any state-of-art methods however requires a large number of labeled instances. In this context our contribution is the proposal of a new method of automatic generation of an object detector with weak manual labeling for the quality control. We evaluate our approach in the task of creating an improved car detector using monitoring cameras in two different climate settings.

The main contribution of this work is a new object detector generation pipeline with some important advantages. It relies on a semi-supervised approach and weak dataset annotation. It explores different weak and strong classifiers to improve generalization. It incorporates a new method for quality control that also relies on weak annotation. And it has been successfully tested using low quality urban cameras available on-line for training and test set formation. As a bonus the full 


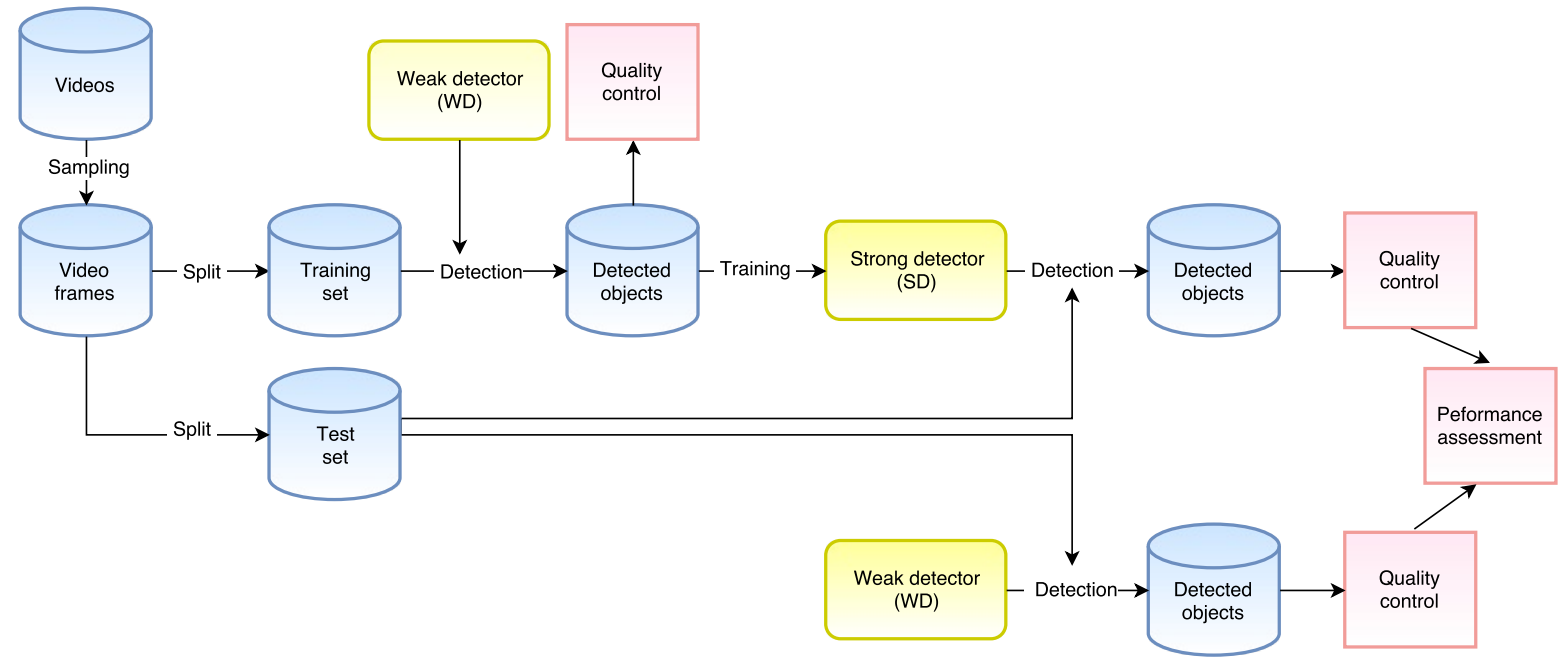

Figure 3.1: Proposed framework. Frames are sampled and split into training and test sets. A weak detection is performed on the training set and used to train a new detector (SD). Quality control is performed both on the detections of the new detector on the test set as well as on the detections of the weak detector on the same set.

dataset used in the experiments is made public ${ }^{1}$.

\subsection{Material and Methods}

The first stage consists in the acquisition of a large set of images and/or videos of interest. Instead of using available datasets, the proposed implementation retrieves data from public monitoring cameras. This solution allows a high variability and amount of data that may be retrieved. This stage included a failure-proof mechanism to take into account issues on the client-side, server-side and on the network. An optional sampling of the videos can be adopted to cope with the redundancy of the objects in consecutive frames if no temporal coherency such as [50] is taken into account.

The DPM method [31] was adopted as our WD. The training set is processed by this approach and the resulting detections are used to train a new RCNN detector, using the classification layers pre-trained on ImageNet [104] (see Figure 3.1).

A critical aspect of the proposed approach is to assure the quality of the intermediate representations obtained by semi-automated ways. A quality control step is performed to evaluate the performance of WD in this task and also to evaluate how better (or not) will the SD be. In the quality control stage we want to estimate the real performance of an object detector given the detected objects in a sample.

In order to evaluate the accuracy of each detector over the dataset, it is desirable to have the proportion $p$ of the true-positives each detector has produced. However, to avoid excessive manual work on annotating the images we estimate the proportion $\hat{p}=T P /(T P+F P)$ over a randomly selected sample from the dataset. The confidence interval for a population proportion $\hat{p}$ based on a sample of size $n$ is given by Equation 3.1 where $z$ has a normal distribution [10]. This strategy allows treating large data sets without a heavy manual burden.

$$
\hat{p} \pm z_{\alpha / 2} \sqrt{\frac{\hat{p}(1-\hat{p})}{n}}
$$

We heuristically choose a $\hat{p}(1-\hat{p})$ value on which outcome we expect from this experiment. The worst case happens when we need the largest $n$ and $\hat{p}=0.5$, since $\hat{p}(1-\hat{p})$ reaches its maximum value. A good practice is to collect a small pilot sample to calculate the proportion over this sample.

\footnotetext{
${ }^{1}$ http://tokudaek.github.io/
} 
We adopted a pilot sample of 50 images to obtain a $\hat{p}(1-\hat{p})=(0.8 \times 0.2)$. Thus, with $95 \%$ confidence $\left(z_{0.025}=1.96\right)$ and a margin of error $\epsilon \approx 2.7 \%$, we used Equation 3.1 to obtain $n \approx 850$.

Instead of fully annotating our training set we annotate the detections instead. We evaluate if each object detected is a match. That way only true positives (TP) and false positives (FP) are obtained and despite the precision on the sample can be directly computed, the recall of the sample is in principle unknown because the number of false negatives is required to compute the recall. Once again, instead of annotating all the dataset for recall, the same approach for estimating the precision can be done by annotating a randomly selected sample.

The performance evaluation of the SD is carried out based on the results of the two quality control stages. We avoid the computation of the recall of each quality control stages by considering just the relative gain in the recall. We use the fact that the number of images tested for both initial detectors, WD and SD, are the same $\left(T P_{W D}+F N_{W D}=T P_{S D}+F N_{S D}\right)$. We can finally compute the relative gain in the recall of $\mathrm{WD}, r g\left(\right.$ recall $\left._{W D}\right)$ defined by Equation 3.2.

$$
\begin{aligned}
& \operatorname{rg}\left(\operatorname{recall}_{W D}\right)=\frac{\text { recall }_{S D}-\text { recall }_{W D}}{\text { recall }_{W D}} \\
& =\frac{\left[\frac{T P_{S D}}{T P_{S D}+F N_{S D}}\right]-\left[\frac{T P_{W D}}{T P_{W D}+F N_{W D}}\right]}{\frac{T P_{W D}}{T P_{W D}+F N_{W D}}} \\
& =\frac{T P_{S D}-T P_{W D}}{T P_{W D}}
\end{aligned}
$$

The quality control stage can be summarized in the following sequence of steps:

1. Compute a sample size, according to Equation 3.1

2. Label a pilot sample

3. Compute TP and FP

4. Compute rg(recall) using Equation 3.2

\subsubsection{Comparative Performance Assessment}

The SD detector is evaluated on the test set and a quality control stage is also performed. The test set is also processed by the WD and quality control is performed in this stage as well. This allows assessing the gain obtained by the strong classifier with regard to the weak classifier. The results of the two quality control stages are combined to infer the accuracy of the SD.

\subsection{Experimental Results and Validation}

We validated the proposed method in two experiments. In the first one, we created a car detector for images from traffic cameras. In the second one we were motivated by a more difficult task: car detection in rainy weather. There are multiple works dealing with the problem of rain removal [146], but few tackling the problem of finding cars in the rain [62]. So in the second experiment we restricted our dataset to images of rainy weather and evaluated our pipeline using this data source. All experiments were performed in a Intel CPU E5-2670 2.30GHz, 256GB RAM with a GeForce Titan X.

\subsubsection{Dataset}

In order to provide an appropriate test bed for our experiments, we have collected 2,327 hours of streaming from public traffic cameras. We utilized [15] to find and filter the cameras. We continuously decoded HTTP live streaming [114] coupled with a relational database to store the metadata. 

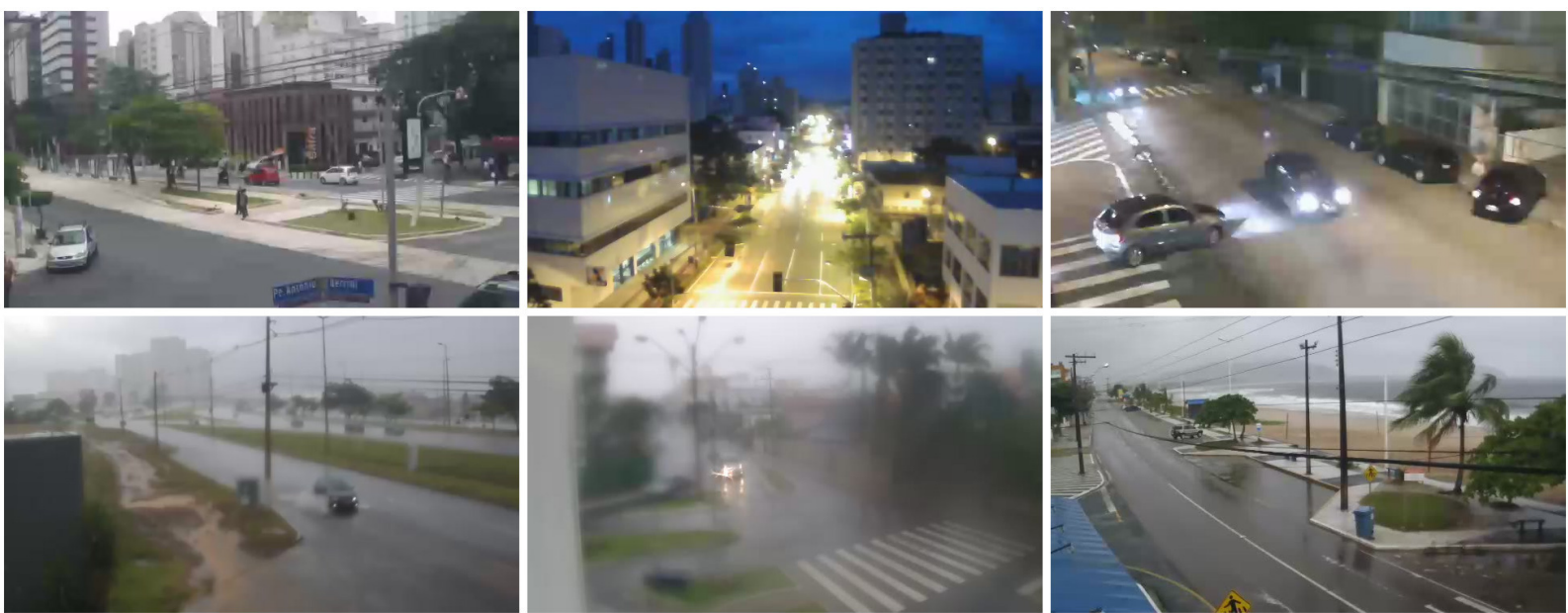

Figure 3.2: Sample of the collected dataset. The first row depicts images from the uncontrolled weather set while the second row shows images from the rainy set.

The videos have 1-minute duration, 640x368 spatial resolution and frame rate of 10fps. The acquisition was divided into uncontrolled weather days and rainy days (see Figure 3.2). From November 30 th to December 3rd, 2015, 41 cameras were continuously monitored, resulting in roughly 2,274 hours of video. The rainy subset was captured from 14 cameras, from March 25th to 26th, 2016 resulting in approximately 53 hours of video.

\subsubsection{Validation 1: Uncontrolled Weather}

In the first experiment, we used the images from the uncontrolled weather subset. The videos were systematically sampled at a $1 / 20$ rate and the 358,036 frames obtained were split into two groups. The training set of 300,000 frames $(\sim 84 \%)$ and the test set of $58,036(\sim 16 \%)$. We applied the DPM [31] on the training set and a quality control step was performed on this stage. The DPM took on average $16.4 \mathrm{~s}$ per image. 557,036 cars were detected and used as the training set of an RCNN detector with a VGG16 architecture [111]. The training process with 10,000 epochs took 27 hours. We evaluated the resulting detector $R C N N_{\text {all }}$ on the test set and a second quality control stage was performed. The quality control results are expressed in Table 3.1.

Table 3.1: Quality control over the Validation 1 and Validation 2.

\begin{tabular}{llcccc}
\hline & Detectors & TP & FP & Precision & Recall \\
\hline \multirow{2}{*}{ Validation 1 } & WD $(D P M)$ & 1366 & 127 & $91.5 \%$ & \\
& SD $\left(R C N N_{\text {all }}\right)$ & 2638 & 345 & $88.4 \%$ & \\
& $r \cdot g \cdot W D$ & & & $-3.2 \%$ & $+93.2 \%$ \\
\hline \multirow{2}{*}{ Validation 2 } & WD $(D P M)$ & 914 & 115 & $88.8 \%$ & \\
& SD $\left(R C N N_{\text {rainy }}\right)$ & 1512 & 449 & $77.1 \%$ & \\
& $r \cdot g \cdot W D$ & & & $-12.5 \%$ & $+65.8 \%$ \\
\hline
\end{tabular}

In the performance assessment of the $R C N N_{\text {all }}$, a relative gain of precision of $\operatorname{rg}\left(\right.$ precision $\left._{W D}\right)=$ $-3.2 \%$ and a relative gain $r g\left(\right.$ recall $\left._{W D}\right)=93.2 \%$ on WD performance was obtained with $95 \%$ confidence level. The generated detector thus presents a significant increase in recall with the trade-off of losing a little precision.

\subsubsection{Validation 2: Rainy Days}

In the second set of experiments, we created a car detector using the rainy subset. Here as well, the raw videos were sampled at a 1/20 rate. The 7,011 frames obtained were split into 6,000 (training 

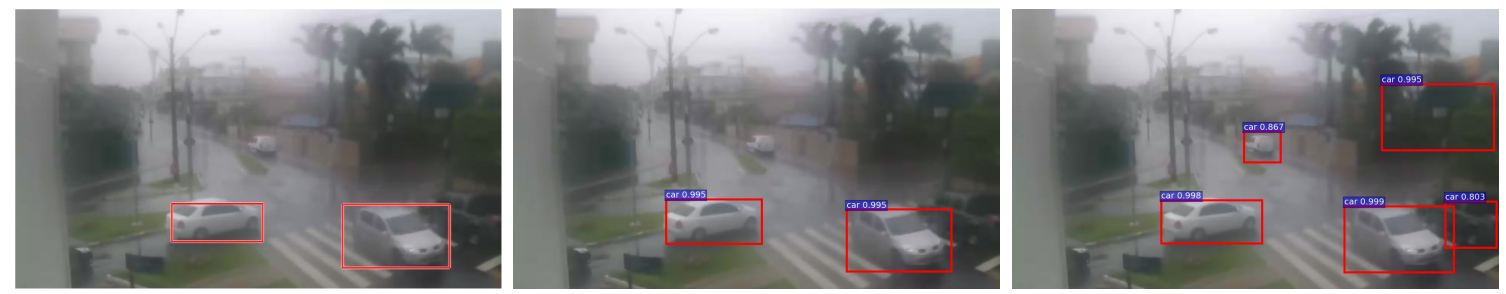

Figure 3.3: Comparison of the cars detections performed by the $D P M, R C N N_{\text {all }}$ and $R C N N_{\text {rainy }}$, respectively, from left to right. The last frame indicates the pros (increased recall with two extra cars detected) and cons (a false positive leading to precision loss) of the proposed approach.

set $\sim 85 \%$ ) and 1,011 (test set $\sim 15 \%$ ). We applied the DPM [31] on the training set and a quality control step was performed on this stage as well. The method took on average 16.8s per image. The 17,325 cars detected were used to fine-tune $R C N N_{\text {all }}$, the detector previously obtained with images from uncontrolled weather. A final detector $R C N N_{\text {rainy }}$ was then obtained after 21 hours. The second quality control stage was then performed. Manual inspection was performed on a sample of 500 images from the test set, according to the quality control step proposed in Section 3.3. The results of the quality control stages are expressed in Table 3.1. In the performance assessment of the $R C N N_{\text {rainy }}$, relative gains $r g\left(\right.$ precision $\left._{W D}\right)=-12.5 \%$ and $\operatorname{rg}\left(\right.$ recall $\left._{W D}\right)=65.8 \%$ were obtained with a $95 \%$ confidence level. Ultimately we obtained a detector with a small loss of precision but high gain in recall in the specific setting of rain and with a simple manual quality assessment. An example is depicted in Figure 3.3.

\subsection{Discussion and Concluding Remarks}

Annotating large amounts of data is challenging. An option is to perform it manually, which is labor-intensive for big data. Alternative options include hiring crowdsourcing services. They may though not suit everyone's needs and new approaches to minimize human operation are desirable (synthetic images if possible [110, 123, ?]). In this scenario, the contribution of this work is the proposal of a methodology for generating object detectors with quality control and minimal manual annotation. The source data is initially processed by a weak detector and the resulting detections generated are used to create a new detector. In both steps, the user inspects a small sample of detections looking just at the true and false positives ratio. We validated our approach with the creation of a car detector using monitoring cameras that was able to produce a relative gain on the precision and on the recall of the weak detector of $-3.2 \%$ and $93.2 \%$, respectively. We performed the same pipeline using rainy images and we got $-12.5 \%$ and $65.8 \%$ variation in precision and recall, respectively. These results show that the strong classifier presents a substantial improvement in recall with a small loss of precision. The full dataset is released. 


\section{Chapter 4}

\section{Impact of de-raining to object detection}

As discussed in the previous chapter, weather events may severely impact scene visibility. It is expected that they do also affect computer vision tasks performances, in particular object detection. In this chapter, the impact of de-raining algorithms on the object detection task using images from low-resolution surveillance cameras is evaluated. The main outcomes of this chapter have been published in [64] ${ }^{1}$, in which this chapter is based on.

\subsection{Introduction}

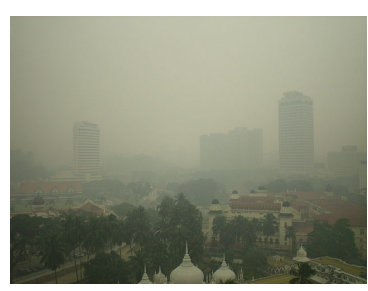

(a) Haze

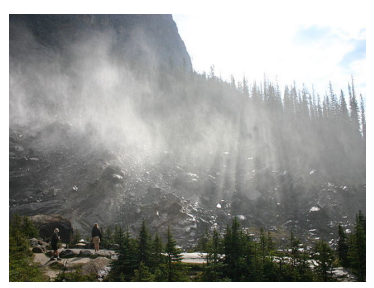

(b) Mist

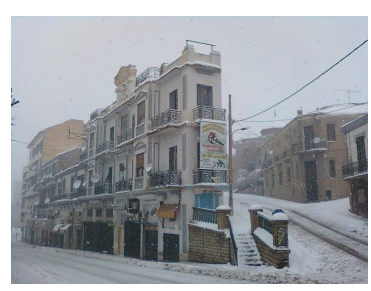

(c) Snow

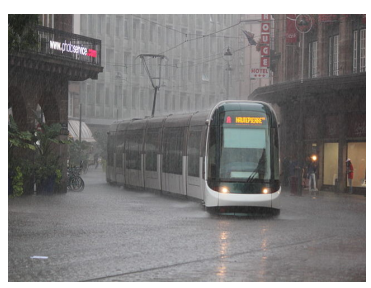

(d) Rain

Figure 4.1: Visual degradation by adverse weather conditions. Images from [138].

Adverse weather conditions such as haze, mist, snow and rain often causes visual degradation due to the interaction of the light and suspended particles as depicted in Figure 4.1. A number of factors affect the visual degradation including distance to the rain and rain intensity. Close raindrops may blur the background or add specular highlights. At intermediate intensities, rain can be similar to parallel streaks. Heavier intensity rain can cause a hail that may completely occlude the scene. Significant effort has been put on the study of rain removal image processing and Chapter 3 presented some experiments that illustrate such challenges for computer vision algorithms. At least two categories of the problem can be elucidated: rain removal from videos and from single images. The problem of de-raining from videos has been extensively explored and the literature reports high accuracies in the task [127]. The analogous task using a single images, single-image rain removal, is considerably more challenging because there is no access to the temporal coherence information between frames present in the former case.

\section{Rain removal}

There is an increasing interest in the problem noticed by the number of publications in the area. The common way to evaluate de-raining images is using reference metrics, such as the traditional Peak Signal-to-Noise-Ratio (PSNR) [46] and Structural Similarity Index (SSIM) [136]. Since the

\footnotetext{
${ }^{1}$ This work has been carried out in a collaboration with professors Siyuan li, Iago Breno Araujo, Wenqi Ren, Zhangyang Wang, Roberto Hirata Junior, Roberto M. Cesar Junior, Jiawan Zhang, Xiaojie Guo and Xiaochun Cao.
} 
rain is synthetically generated, they can measure how much the method was able to recover the target image. Alternatively, there are metrics that try to evaluate the perceptual quality of the images with no clean image for reference. Three well-known human perceptual quality assessment metrics are Naturalness Image Quality Evaluator (NIQE) [82], Spatial-Spectral Entropy-based Quality (SSEQ) [71], and Blind image Integrity INdicator using DCT statistics (BLIINDS-II) [105]. NIQE is a popular no-reference image quality metric to indicate that attempts to measure the naturalness of an image. The smaller the score with such metric indicates better image quality. NIQE utilizes both spatial and spectral information of the target image as features to learn and predict the image quality. BLINDS-II also applies ML techniques to learn the image quality, but they use as features discrete cosine transform features.

Various methods have been proposed to solve the problem of single-image rain removal. Pioneer works incorporated concepts such as sparse coding [76], Gaussian mixture models [67], and low-rank representation [19]. The majority of the recent works on the problem, though are DL-based. Five state-of-the-art DL-based methods are described below.

In the Joint Rain Detection and Removal (JORDER) [141], the authors argue that the spatial contextual information is helpful in rain removal and thus, they propose a DL-based approach with enlarged receptive fields. The proposed network jointly performs detection and rain removal and is put inside a recurrent framework to overcome the mutual presence of different rain effects such as rain accumulation and diverse rain streaks intensities and orientations.

In [33] the authors observe that the network proposed by [41] with slight modifications achieve good results in the task of single-image rain removal, particularly in the presence of heavy intensity rain. So they modify the network proposed by [41] and propose propose the Deep Detail Networks (DDN) which considers a layer of the image composed of the objects and the rain in the image, i.e., without the background.

In the [145], the Image De-raining Conditional Generative Adversarial Network (ID-CGAN) is proposed. The proposed generative adversarial network is composed of discriminative model that discriminates between a real and a fake image and a generator model that aims to translate the input rainy image to clean images constrained by the discriminator definition of clean and rainy image. To mitigate the GANs generation of artifacts and difficulties to train, the authors introduce a novel loss including a term that minimizes the perceptual difference between reconstructed and the ground-truth image.

In the Density-aware Image De-raining Using a Multi-stream Dense Network (DID-MDN) [144] the main assumption is that if information about the rain intensity is known, them a more tuned rain streak removal can be utilized. They leverage the task by identifying the level of rain density (light, medium or heavy) and removing the rain using a model of rain streaks according the identified density. They utilize an additive model of rain and streaks and they estimate the rain component (residual) using a CNN-based classifier.

In [95], the authors also utilize generative adversarial networks, but differently from the works above, the authors focus on the problem of raindrops removal, not necessarily from rain. Their method, here called DeRaindrop, is a generative adversarial network composed of a discriminator that is able to discern between real and unrealistic images and the generator, that is responsible for removing the raindrops from the affected image. The generator is the most complex part, and it is composed of visual attention map generation and the localized removal of the raindrops.

\section{Challenges}

The literature covers a number of works on rain streaks removal and the reported state-of-theart results achieve high values. In the same way, raindrops removal methods are able to effectively improve the visibility of the image. However, at least three limitations of these works can be mentioned: they perform shallow experiments or even disregard experiments on the impact on computer vision tasks; and the assumption that rain is modeled by visible rain streaks or raindrops is very strong and possibly limiting; they perform experiments with high-quality images. In the special case of images from surveillance cameras, images may present low-resolution and additional environmen- 
tal challenges such as jittering and illumination and they may be integrated into larger automated environments. In this scenario, the impact on computer vision tasks can be more important than the perceptual improvements on the image. In this practical setting, one may ask:

Question 2: Can the current single image de-raining methods help in the task of object detection in low-resolution images in rainy weather?

The contributions described in this chapter can be summarized as (1) a benchmark of state-ofthe-art methods of single image de-raining coupled with state-of-the-art object detectors to analyze the change in performance and (2) the creation of a repository of images from surveillance cameras in rainy weather. The experiments show that no current de-raining method was able to help the evaluated object detection methods using images from surveillance cameras.

\subsection{Proposed Evaluation Protocol}

An overview of the protocol can be visualized in Figure 4.2. Images were systematically acquired from surveillance cameras from the internet in form of streaming. Frames were extracted with a minimum offset of 250 between each frame. A typical scene depicts the street, vehicles and pedestrians. Images were manually filtered considering a diverse and daytime set of rainy images from surveillance cameras.

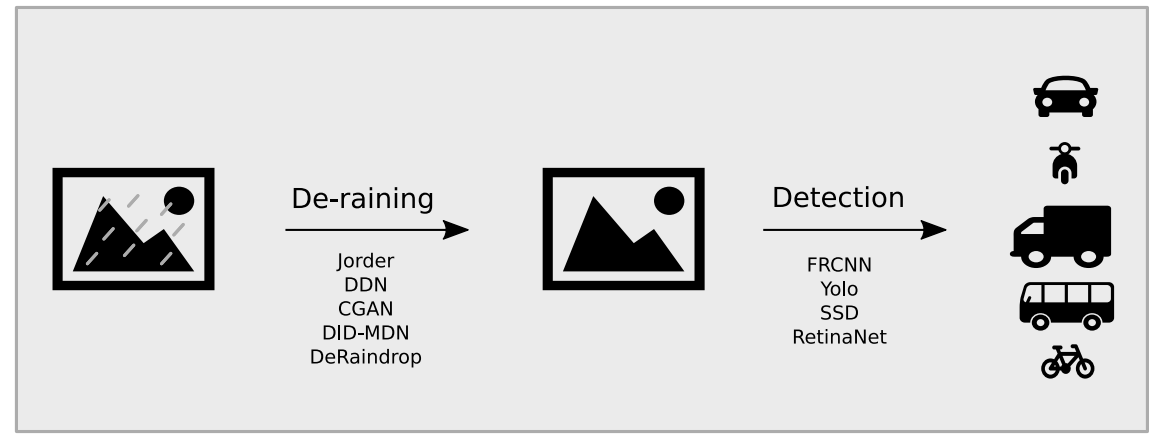

Figure 4.2: Evaluation protocol. The surveillance images from rainy weather are processed using one of the five de-raining algorithms and is processed by one of the 4 object detection algorithms.

Since there is no access to ground-truth for real rainy images, three no-reference image quality assessment metrics were utilized: NIQE [82], SSEQ [71] and BLIINDS-II) [105]. The main object categories present in the dataset consist of vehicles, buses, cyclists, bikers and trucks. Remaining categories showed low occurrences and was disregarded. All objects of interest were manually annotated in the dataset.

Five de-raining algorithms were chosen based on the reported results and on the code availability: JORDER [141], DDN [33], CGAN [145], DID-MDN [144] and DeRaindrop [95]. They are independently applied over the dataset and no-reference metrics are computed. Full reference metrics were not computed given the impossibility of computing the degradation of the rain in isolation.

Four state-of-the-art object detection algorithms were considered: FRCNN [100], YOLO-V3 [99], SSD-512 [72] and RetinaNet [69]. Each method is explained in Chapter 2. Each object detection method performance was evaluated considering the average precision metric as defined in [30].

\subsection{Experimental Results}

The results of the experiments are detailed in this section. A total of 2,048 images from a total of 154 cameras have been obtained. Image resolutions are of $640 \times 368$ or of $640 \times 480$ in compressed JPEG format. The objects were manually annotated and the objects are expressed in Table 4.1. It can be seen the prevalence of vehicles with a few number of motorcycles. Such numbers are 
probably influenced by that fact it was raining at the time of the image acquisition (fewer cyclists and pedestrians).

Table 4.1: Number of objects per category.

\begin{tabular}{ccccc}
\hline Car & Person & Bus & Truck & Motorcycle \\
11,415 & 2,687 & 488 & 673 & 275 \\
\hline
\end{tabular}

Each de-raining method is independently applied on the whole set and a new set of de-rained images is generated for each method. No-reference metrics were computed using this new set and they have been summarized in Table 4.2. According to these metrics, in fact there are some improvements on the image generated, despite they do not agree on which method improved most.

Table 4.2: Average no-reference evaluation results after de-raining processing.

\begin{tabular}{lcccccc}
\hline & Rainy (raw) & JORDER & DDN & ID-CGAN & DID-MDN & DeRaindrop \\
\cline { 2 - 7 } SSEQ & 48.24 & $\mathbf{5 4 . 8 8}$ & 53.47 & 53.36 & 54.64 & 54.28 \\
NIQE & 6.45 & 6.12 & 6.29 & $\mathbf{5 . 0 3}$ & 5.42 & 5.81 \\
BLINDS2 & 54.89 & 58.78 & 57.52 & 60.86 & 57.92 & $\mathbf{6 9 . 0 6}$ \\
\hline
\end{tabular}

The de-rained images are processed by each of the four object detection algorithms. Results are expressed in Table 4.3. The object detection methods accuracies have high variance between categories and between themselves. However, there is little variance in the accuracies among the de-raining methods and they invariably degrade the detection performance with a few exceptions such as from the detection of trucks by SSD. See Figure 4.3 for a representative example (more examples can be seen in Appendix B). This fact goes against what indicates the no-reference metrics in Table 4.2 but that is not a surprising feature given that these metrics were not proposed with this goal in mind.

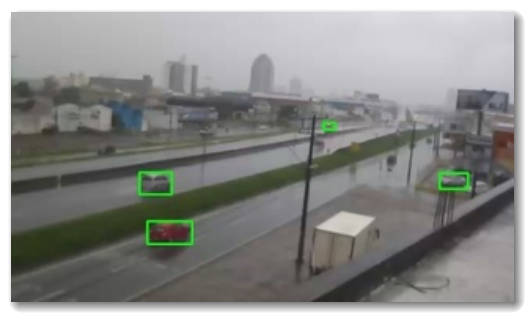

(a) Rainy (raw)

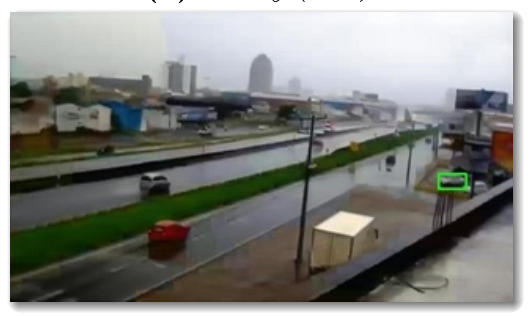

(d) $I D-C G A N$

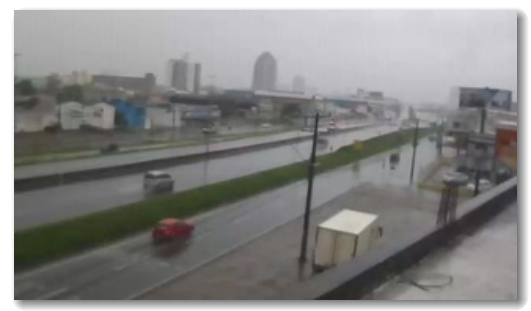

(b) JORDER

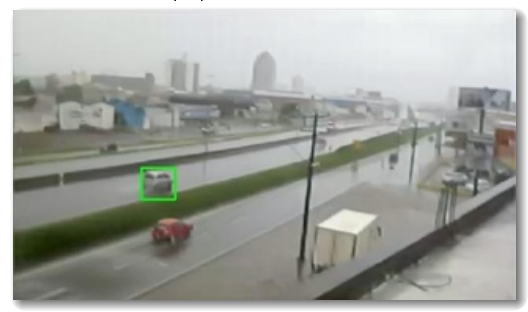

(e) $D I D-M D N$

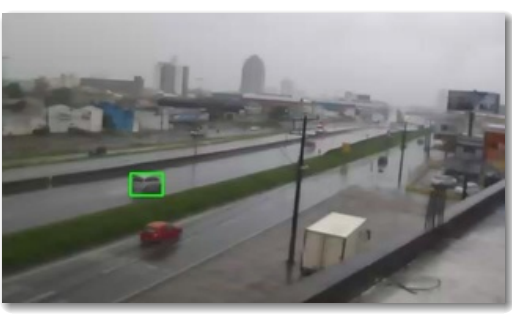

(c) $D D N$

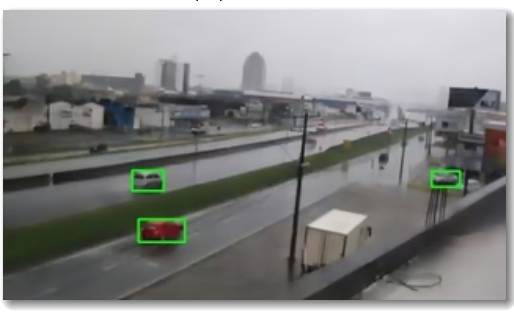

(f) DerainDrop

Figure 4.3: Example of detections of YOLO-V3 in the rain. Figure (a) corresponds to the detection without pre-processing, while $(b),(c),(d),(e),(f)$ correspond to the detections with images pre-processed by JORDER, $D D N, D I D-M D N$, DerainDrop, respectively. 
Table 4.3: Detection results per category and per de-raining method. Horizontal groups correspond to categories and to the mAP (bottommost category). Each cell corresponds to a pre-processing methods (de-raining) column and to a detection method row. The pre-processing methods include Rainy (raw images), JORDER, DDN, ID-CGAN, DID-MDN and DeRaindrop and the detection methods are FRCNN, YOLO-V3, SSD-512 and RetinaNet.

\begin{tabular}{clcccccc}
\hline \multirow{5}{*}{ Person } & & Rainy & JORDER & DDN & ID-CGAN & DID-MDN & DeRaindrop \\
\hline \multirow{5}{*}{ Truck } & FRCNN & 14.65 & 14.51 & 14.02 & 10.69 & 11.37 & 12.77 \\
& YOLO-V3 & 21.25 & 20.18 & 20.33 & 15.18 & 18.43 & 16.73 \\
& SSD-512 & 13.05 & 11.78 & 11.33 & 8.32 & 11.80 & 9.70 \\
& RetinaNet & 10.59 & 8.81 & 8.15 & 6.22 & 7.22 & 8.61 \\
\hline \multirow{5}{*}{ Car } & FRCNN & 20.29 & 17.71 & 17.83 & 14.14 & 17.31 & 17.23 \\
& YOLO-V3 & 21.25 & 11.80 & 12.51 & 9.48 & 12.75 & 12.52 \\
& SSD-512 & 3.06 & 3.95 & 3.76 & 2.92 & 4.15 & 3.88 \\
& RetinaNet & 8.14 & 6.45 & 6.06 & 4.66 & 6.78 & 7.68 \\
\hline \multirow{5}{*}{ Bus } & FRCNN & 38.54 & 38.00 & 37.54 & 35.80 & 36.37 & 35.80 \\
& YOLO-V3 & 44.93 & 40.21 & 43.23 & 42.07 & 42.61 & 42.39 \\
& SSD-512 & 20.87 & 19.60 & 21.42 & 21.36 & 20.25 & 20.39 \\
& RetinaNet & 28.80 & 26.19 & 26.36 & 26.26 & 25.31 & 25.20 \\
\hline \multirow{5}{*}{ Motorbike } & FRCNN & 29.85 & 26.77 & 25.41 & 22.08 & 23.28 & 27.04 \\
& YOLO-V3 & 29.63 & 25.40 & 25.55 & 21.85 & 27.79 & 25.87 \\
& SSD-512 & 1.96 & 2.29 & 2.24 & 2.01 & 1.87 & 3.79 \\
& RetinaNet & 11.29 & 8.67 & 7.88 & 6.30 & 9.04 & 9.10 \\
\hline & FRCNN & 10.06 & 10.07 & 9.02 & 7.41 & 6.29 & 7.00 \\
& YOLO-V3 & 6.92 & 4.64 & 7.39 & 4.97 & 5.90 & 4.64 \\
& SSD-512 & 2.02 & 2.08 & 2.69 & 0.91 & 2.96 & 2.89 \\
& RetinaNet & 5.25 & 3.44 & 3.49 & 3.37 & 3.29 & 3.67 \\
\hline \multirow{6}{*}{ mAP } & FRCNN & 22.68 & 21.41 & 20.76 & 18.02 & 18.93 & 19.97 \\
& YOLO-V3 & 23.27 & 20.45 & 21.80 & 18.71 & 21.50 & 20.43 \\
& SSD-512 & 8.19 & 7.94 & 8.29 & 7.10 & 8.21 & 8.13 \\
& RetinaNet & 12.81 & 10.71 & 10.39 & 9.36 & 10.33 & 10.85 \\
\hline
\end{tabular}

\subsection{Conclusion}

In this part of the thesis, the impact of the de-raining algorithms to the object detection performance using low-resolution images from surveillance cameras was assessed. For this purpose, object detection was preceded by a de-raining processing step. Five state-of-the-art de-raining methods and four object-detection methods were utilized using this procedure to identify five different object categories from a set of approximately 2,000 real rainy images. Unexpectedly, none of the utilized de-raining methods showed overall improvement on the detection task. Given the limitation of having tested on a subset of all available de-raining and object detection methods, these results cannot assertively affirm that current de-raining methods do not help on the detection task when using low-resolution surveillance images, but it is a strong indicator of so. This discovery may encourage the community to create de-raining or object detection methods that can handle the particular type of images utilized in this work. 


\section{Chapter 5}

\section{Inaccurate Measurements in Sensor Networks}

\section{$5.1 \quad$ Introduction}

Pedestrians represent an integral and pervasive aspect of the urban environment. Real estate, consumer patterns, public safety, and other aspects of city life are deeply intertwined with the variations of distribution of people across a city. However, current methods for estimating the distribution of people within a city tend to be expensive and mostly produce a sparse sampling of a few locations.

In this chapter we propose a novel method to estimate the pedestrians distribution using streetlevel pictures of the city. We determine bounds for the estimate and apply the methodology using a large and rich collection of street-level pictures from New York city to obtain a dense pedestrian map of the region.

We make use of state-of-art computer vision methods to identify and count pedestrians and we take into account the errors inherent to the detection process, we model it as a probabilistic detection and provide a closed form and bounds for the asymptotic error of the sampling process. We compare these formulas to numerical simulations of the sensing process. Our results suggests that computer vision produces usable data, despite the inherent noise. We utilized over 40 million street-level images provided by Carmera ${ }^{1}$. These images were obtained via their partnerships with high coverage fleets operating daily on city streets that traveled through the region of Manhattan Island in New York City over the course of a year. A deep-learning based algorithm for pedestrian detection was utilized in a case study to map the density of pedestrians in Manhattan.

The contributions of this chapter can be summarized as

1. A new method for the analysis of the distribution of people using state of the art, but imperfect, computer vision algorithms.

2. A closed form function and bounds for the asymptotic error of the resulting person densities.

3. The results of simulations validating the sampling process and the derived asymptotic error.

4. A case study demonstrating the resulting densities for a collection of images from the City of New York.

This work has been accepted in ACM TSAS [122] in which this Chapter is based on ${ }^{2}$

\footnotetext{
${ }^{1}$ http://www.carmera.com

${ }^{2}$ This work has been carried out in a collaboration with Claudio T. Silva, Roberto M. Cesar-Junior, Yitzchak Lockerman, Gabriel Ferreira, Ethan Sorrelgreen and David Boyle.
} 


\subsubsection{Related Work}

There are many ongoing efforts on the use of urban data to achieve citizen-centered improvements [147]. Governments and organizations in urban environments collect a vast amount of data daily [130] encompassing a large assortment of information including mobility, crime and pollution. The collection and use of this information has been attracting attention from the academics, governments and corporations [132]. The work [7] explores the correlation of visual appearance of pictures and the attributes of the region it pertains. They collected images from [38] and also indicators from multiple regions and trained a model [14] to predict the indicator based on images. The city attributes include violent crime rates, theft rates, housing prices, population density and trees presence. Results show that the visual data can be efficiently used to predict the region attributes. Additionally, the regressor trained in one region showed reasonable results when tested in a different city. In a different problem, Zemene et al. [143] proposes a technique of determining the geo-location of the scene depicted in an image. Local and global features are extracted and used in a posterior features matching step. They report high accuracy and speed performances compared to existing approaches.

A person map of the city has numerous applications for urban planners including the design of public transport network and of public spaces [137]. One approach to obtain a citywide count of person is to have people scattered around the city manually counting the person nearby. This approach though is laborious because it requires dedicated people to perform the measures. Another possibility explored in [97] is to use cellphone use data to perform the person count. One clear limitation of this approach is that these data are not public and their coverage are restricted to the places where the carrier signal is present. Additionally, it is hard to know whether the cell signal is from a person or from someone in a building or from someone in a car.

Alternatively, we can consider the visual task of finding people in city images. A remarkable work in this task consists in using the histogram of oriented gradients as the features vector and a support vector machines for the classification task [26]. In the context of deep neural networks [55, 115], the work of [100] introduced an approach that tries to solve this task by using a unified network that performs region proposal and classification. In this way, the method accepts annotations of multiple sized objects during the training step and during the testing stage, it performs classification of those objects in images of arbitrary sizes. In [25] the authors follow the two-stage region proposal and classification framework of [100] and proposes the Region-based Fully Convolutional Networks (R-FCN) which incorporate the idea of position-sensitive score maps to reduce the computational burden by sharing the per-RoI computation. Such speed alterations allow the incorporation of classification backbones such as [41]. Alternatively to the traditional person detection, head detection $[35,106]$ approaches have also been explored for efficient person count estimates. Given that information from the body is disregarded, however, it is natural to expect an inferior accuracy in complex environments.

There are several city images repositories that contemplate people, some of them obtained using static cameras [133, 87] and others obtained using dynamic ones [36, 22, 77]. Such configuration of sensors arrangement have long been studied in the sensor network field [1, 2, 90] and an important aspect of these networks is whether the sensors are static or mobile. In [135] the authors explore the setting of a network composed of both static sensors and of mobile sensors. The holes in the coverage of the static sensors network are identified and the mobile sensors are used to cover the holes. A common problem in sensor networks is the $k$-coverage problem defined in [44], that aims to find the optimal setting of sensors such that any region is covered at least by k sensors. In [140] the authors perform the task of counting people based on images obtained through a wireless network of static sensors.

Apart from controllable mobile sensors network, many works explore data collected from collaborative uncontrolled sensors [11] such as from vehicles GPS [109, 51], mobile phones sensors [108, $57,96]$ and even from on-body sensors [21].

The work of [65] considers the problem of using GPS data from a network of uncontrolled sensors to reconstruct the traffic in a city. They do that in two steps: initial traffic reconstruction 
and dynamic data completion. Such approach allowed the authors to get a complete traffic map and a $2 \mathrm{D}$ visualization of the traffic.

There are many ways to model the movement of mobile nodes in a sensor network, the so-called mobility models [16]. A simple one is the random walk mobility model [27] where at each instant in time each particles gets a direction and a speed to move. In the random waypoint mobility model [49], in turn, particles are given destinies and speeds. They travel toward their goal and once they get the destination a new goal and speed are given. The Gauss-Markov mobility model [68] attempts to eliminate abrupt stops and sharp turns present in the random waypoint mobility model. It is done by computing the current position based on the previous position, speed and direction.

Simulation of wireless sensor networks has long been studied [134, 61, 85] because it allows a complete analysis of system architectures by providing a controlled environment for the system [119]. The real-life systems non-determinism is simulated by the use of pseudo random number generators [53]. Among the large number of pseudo random number generators [91], a popular algorithm is the Mersenne Twister [78] due to its efficiency and robustness.

\subsection{Data and Proposed Method}

\subsubsection{Data}

The images utilized in this work come from a fleet of camera equipped cars (similar to [60]) traveling through Manhattan. Carmera provides a temporally and spatially dense collection of pictures. The volume of the sample utilized is at least an order of magnitude higher compared to the most popular images datasets (see Table 5.1). It also differs from urban image databases [38, $133,87,36,22,77]$ by providing dense temporal coverage in addition to dense spatial coverage. The orientation of the cameras varies and the nature of the images are similar to street level collections provided by many mapping services. However, the images are not stitched into a 360 degree panorama. Every image is accompanied by metadata including the acquisition time, location, and camera orientation. The images are captured as the vehicle travels, with no control of the content, the illumination, the weather, the traffic conditions, or vehicular speed. The typical image depicts a urban scenario as a background and the city dynamics including person, vehicles and bicycles.

Table 5.1: Comparison of the numbers of images utilized to other popular datasets.

\begin{tabular}{lcc}
\hline Datasets & Number of images & Resolution \\
\hline CIFAR 10 [54] & $6.0 \cdot 10^{3}$ & low \\
Pascal VOC 2007 [30] & $9.9 \cdot 10^{3}$ & middle \\
Cityscapes [22] & $2.0 \cdot 10^{4}$ & very high \\
MS COCO [70] & $3.3 \cdot 10^{5}$ & high \\
ILSVRC 2017 [104] & $1.2 \cdot 10^{6}$ & high \\
Our sample & $1.0 \cdot 10^{7}$ & high \\
\hline
\end{tabular}

All images included in the sample have a resolution of $1280 \times 960$. We used a sample of images captured from March 2016 to February 2017 containing 10,708,953 images. This sample presents a dense spatial sampling of the whole region of Manhattan (see Figure 5.2) over a year and irregular spatio-temporal sampling on a daily basis (see Figure 5.1) All resulting heatmaps are weighted sampling according to this distribution. Despite the large scale of the collection utilized in this work, it represents a small portion of the full dataset obtained by the company. 

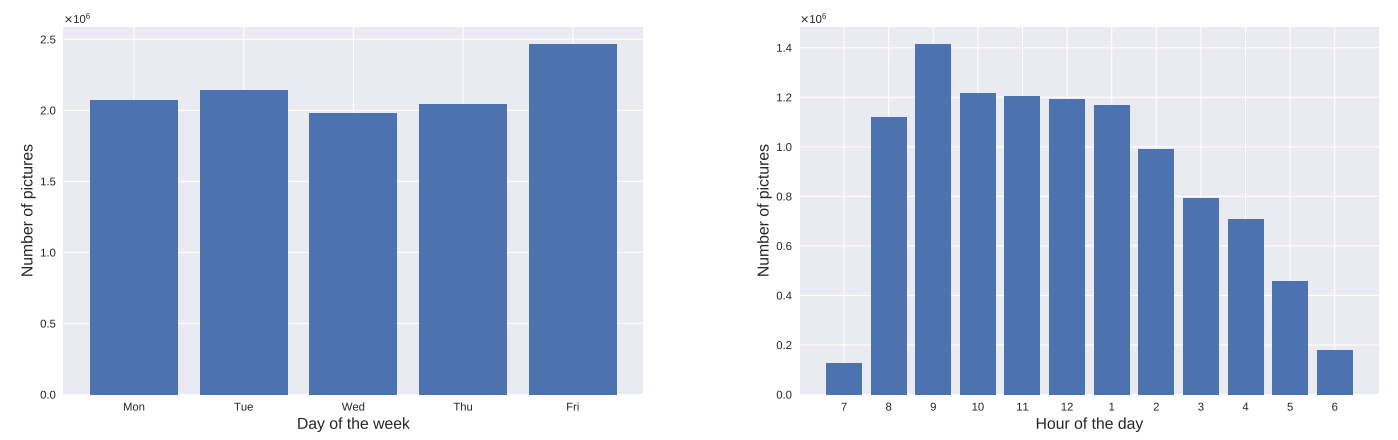

Figure 5.1: Temporal distribution of the collection of images by day of the week (left) and by hour of the day (right).

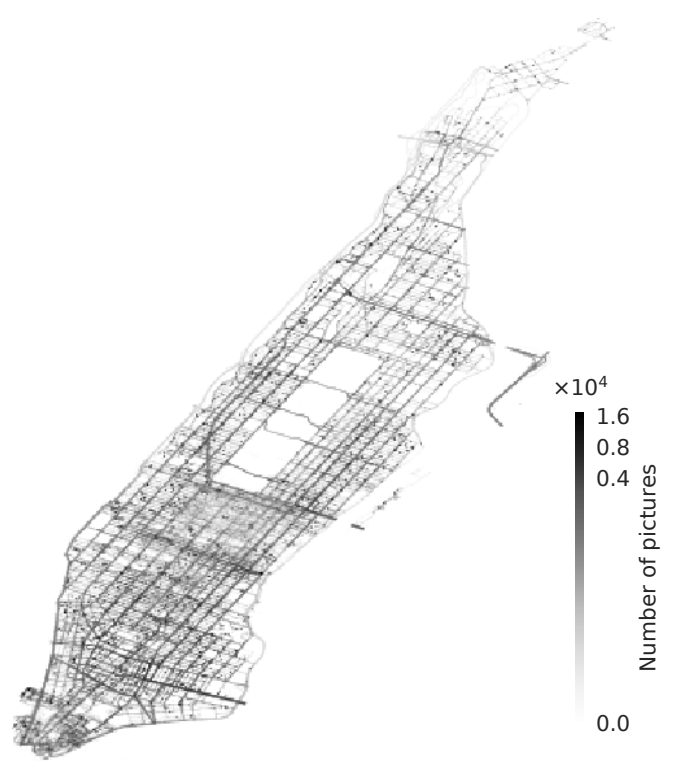

Figure 5.2: Geographical distribution of the number of pictures utilized in the region of Manhattan. The highest concentration of images are in the bottom and middle regions of map. 


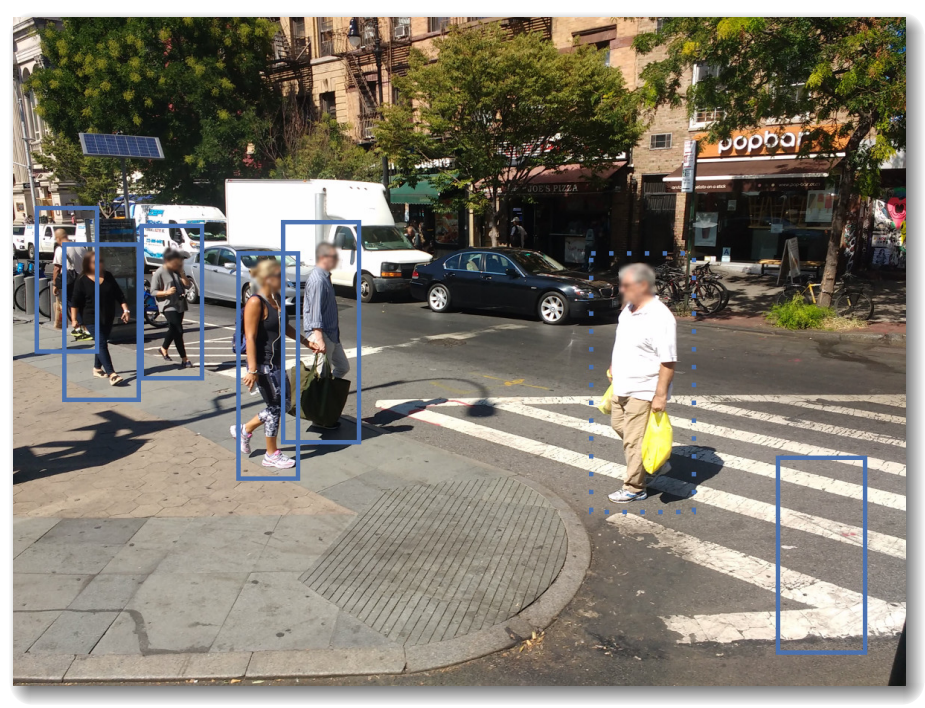

Figure 5.3: Hypothetical person detection. The person on the center was not identified by the detector and is a false-negative. The rightmost detection is a false-positive. Underlying image provided by Carmera.

\subsubsection{Person Detection}

We adopt the same metric as Everingham et al. [30] when comparing the detected objects in an image to the ground-truth. A detected object is considered to correspond to a particular ground truth object if there is a minimum ratio of $50 \%$ between the overlap of the detected bounding boxes $B_{\text {detected }}$ ground-truth bounding boxes $B_{\text {gtruth }}$, and the union of the two areas (see Equation 5.1).

$$
\frac{\left|B_{\text {detected }} \cap B_{\text {gtruth }}\right|}{\left|B_{\text {detected }} \cup B_{\text {gtruth }}\right|}>=0.5
$$

The recognition of distant objects in an image is difficult for humans and is even more difficult for computers. We assume that, on average, the size of a person within an image is an indicator of the distance that person to the sensor and try improve accuracy by considering a minimal size of the people detected. Thus, bounding boxes smaller than a parameter threshold are ignored. Another difficulty regards the differentiation among real people, cyclists and disabled people. The tested methods detect people even in the case they are sitting or riding.

We utilized the deep-learning based method R-FCN [25] over the entire dataset to obtain a database with the number of person detected in each image. This database is then aggregated in space and time to create a visualization of the person counts by finding the average number of person per image in each region.

\subsubsection{Sensing Model}

As current person detection algorithms are far from perfect, it is natural to wonder about the accuracy of any person count resulting from their use. In this section we provide a theoretical analysis of the effect of algorithmic errors on the final count.

In our model, we assume that the world is modeled by a number of small regions, or buckets, each of which we intend to measure a density. Sensors and people move around a world in some random fashion. At regular intervals, each sensor takes an independent measurement of the nearby person count and updates the recorded density at its current location, $x$. More formally, each time a sensor takes a sample, it obtains a measurement represented by the random variable $N(x)$. While we do not specify the distribution of $N(x)$, we assume that the expected value follows the formula

$$
E\left[N_{i}(x)\right]=p n_{i}(x)+\lambda
$$

Here $n_{i}(x)$ is the actual number of people in the location and time being sensed. $p$ is a number 
giving the success rate of the vision algorithm and $\lambda$ indicating its false positive rate.

The result of this process is the density of people at each location, $\psi(x)$.

$$
\psi(x)=\frac{1}{k} \sum_{i} N_{i}(x)
$$

For comparison, the ground truth density $\phi(x)$, defined respectively by (where $k$ is the number of steps and samples),

$$
\phi(x)=\frac{1}{k} \sum_{i} n_{i}(x)
$$

We show in Appendix A.1, Equation A.5 that the expected value of $\psi(x)$ is

$$
E[\psi(x)]=p \phi(x)+\lambda
$$

In other words, $\psi(x)$ is a biased estimator of $\phi(x)$. Unless our sensing algorithm precisely follows Equation 5.5, we are unable to transform this biased estimator into an unbiased one. Furthermore, even in the ideal case, $p$ and $\lambda$ may not be known. Instead, we directly utilize $\psi(x)$ and attempt to find a relative histogram. That is, we expect to get a number proportional to the density of the number of people at a location and not the actual density. As such, for any constant $a$, our density is equivalent to one scaled to $\psi^{\prime}(x)=a \psi(x)$. Treating the distribution as a vector, we measure the direction but not the magnitude. In the terminology of group theory, our measurement suggests a density within the equivalent class:

$$
[\psi]=\left\{a \in \mathbb{R}_{+} \mid a \psi\right\}
$$

To validate our measurement we need a metric that indicates how well the equivalent class compares to the ground truth distribution $\phi(x)$. To do that, we compare the ground truth to the unique closest element within the equivalent class. As a vector projection, this minimum element is (see Appendix A for a proof):

$$
\psi^{\prime}= \begin{cases}\psi \frac{<\psi, \phi>}{|\psi|^{2}} & |\psi| \neq 0 \\ 0 & |\psi|=0\end{cases}
$$

which we can then compare using the usual euclidean metric $\left|\psi^{\prime}-\phi\right|$. However, this metric depends on the number of locations in the map, as well as the number of people. As such, we normalize the metric to between 0 and 1 , to obtain a final metric:

$$
\frac{\left|\psi^{\prime}-\phi\right|}{\left|\psi^{\prime}\right|+|\phi|}
$$

We consider $h>0$ as the average density of people and $c \geq 1$ as the distribution of $\phi . c$ can best be thought of as parameters that describe the asymptotic error. Both of these parameters depend on the resolution of the heat map in addition to person distribution. In many cases $c$ can not be determined, as such we can use the inequality in Equation A.15 of Appendix A.1 to conclude that over long periods of time:

$$
\lim _{k \rightarrow \infty} \frac{\left|\psi^{\prime}-\phi\right|}{\left|\psi^{\prime}\right|+|\phi|} \leq \frac{\sqrt{c^{2}-1} \lambda}{2 c^{2} h p} \leq \frac{1}{4} \frac{\lambda}{h p}
$$

It is important to note that $h$ represents the ground truth density of people, in the same units of $\phi$. If only the sampled average density, $\hat{h}$, is know, the unbiased estimator of $h, \frac{\hat{h}-\lambda}{p}$ can be used. This leads to the bounds

$$
\frac{1}{4} \frac{\lambda}{h p} \approx \frac{1}{4} \frac{\lambda}{\bar{h}-\lambda}
$$



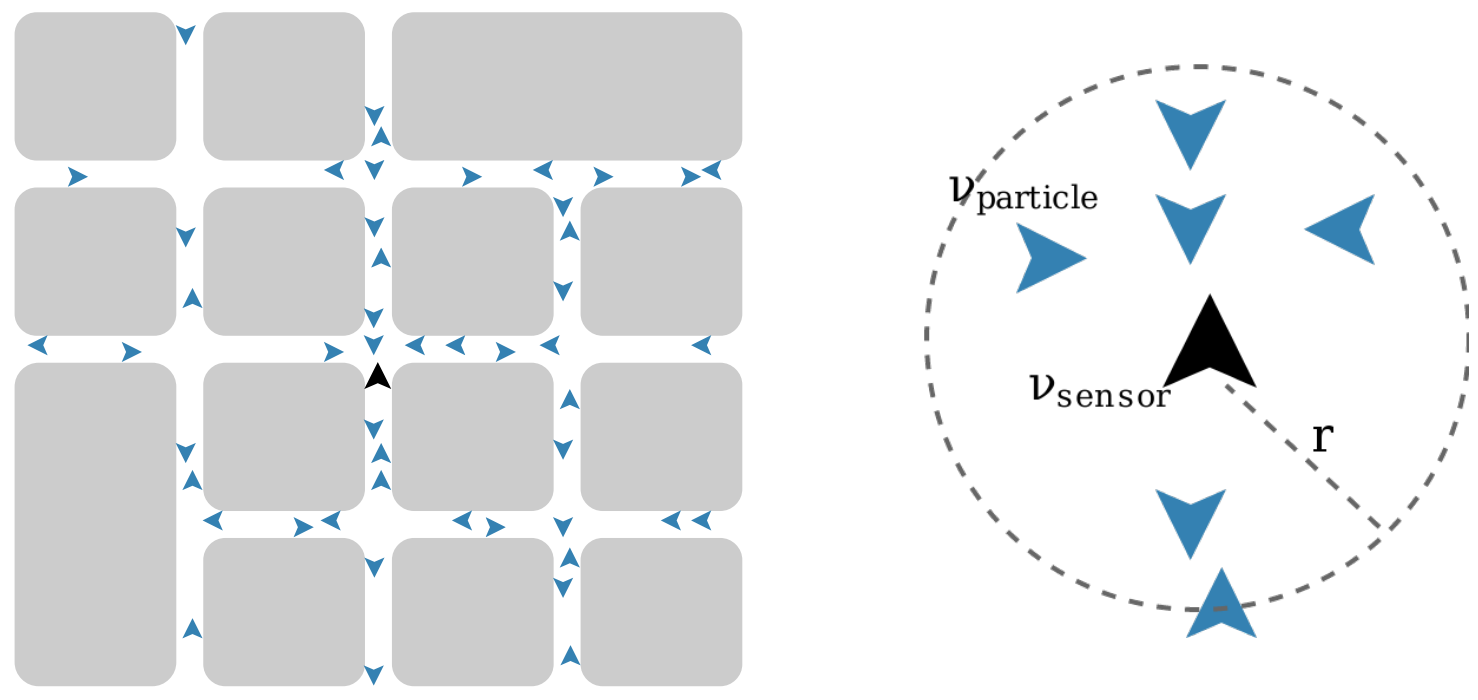

Figure 5.4: Left: An illustration of our simulation containing a sensor (center) moving through an environment with numerous person. Right: Each sensor moves with uniform speed $\nu_{s}$ and is able to sense people within a radius of $r$. Each sensing operation has a probability $p$ of correctly detecting each person and, on expectation, finds $\lambda$ false positives. People move with uniform speed $\nu_{p}$.

This final formula is only dependent on the false positive rate of the sensing algorithm and the average density of sensed objects measured by process, making it suitable for practical sensing applications. We wish to emphasize that this inequality is true whenever Equation 5.5 holds regardless of the underling probability distribution. This function is only useful when $\lambda \leq \bar{h}$ and in that domain, it is a monotonically increasing function of $\lambda$.

\subsubsection{Simulation}

The real-life acquisition process lacks some of the simplifications we used in our model. For example, samples taken in spatial and temporal proximity are correlated. To examine the performance of the sensing systems in the face of these non-ideal circumstances, we created a discrete event simulation [58] to compare sensed distributions to a known ground truths.

As illustrated in Figure 5.4, we simulated a number of mobile sensors that detect nearby particles. Sensors simulate the person detection event and their movement simulate the movement of the camera-equipped fleets responsible for the acquisition of data. Each sensor has a circular coverage of radius $r$. Collision among particles and sensors are ignored for simplicity. Sensors and particles move with uniform speeds $\nu_{\text {sensor }}$ and $\nu_{\text {particle }}$ respectively. The simulation world is mapped as a graph, as in [118]. Each node in the graph is a traversable point by both sensors and particles and edges represent a path between the end nodes.

We assume that, in each time step, sensor has an independent chance, $p$, of detecting each of the $n(x)$ person within range along with an independent chance per location to obtain a false positive. These assumptions lead to $N(x)$ being sampled from the sum of a binomial distribution with mean $p$ and a Poisson process with a given expected number $\lambda$. A calculation of the expected value indicates that Equation 5.2 is satisfied and within the proposed bounds.

The system state can be described by various state variables: sensors and particles positions, sensors and particles waypoints, real density of particles and sensed density of particles. Sensors and particles move with a variation of the random waypoint model [49], differing to it by the fact that sensors and particles are not allowed to change speeds; they have fixed speed given by the system parameters $\nu_{\text {sensor }}$ and $\nu_{\text {particle }}$. When a new destination is randomly picked, the trajectory on the map graph is computed using the $\mathrm{A}^{*}$ algorithm [39] and the points of the trajectory are pushed to a heap (please refer to Algorithm 1).

As time progresses we obtain a 2D histogram for the sensed density as well as the ground truth density of particles. We are primarily interested in the difference between them, as given by the 

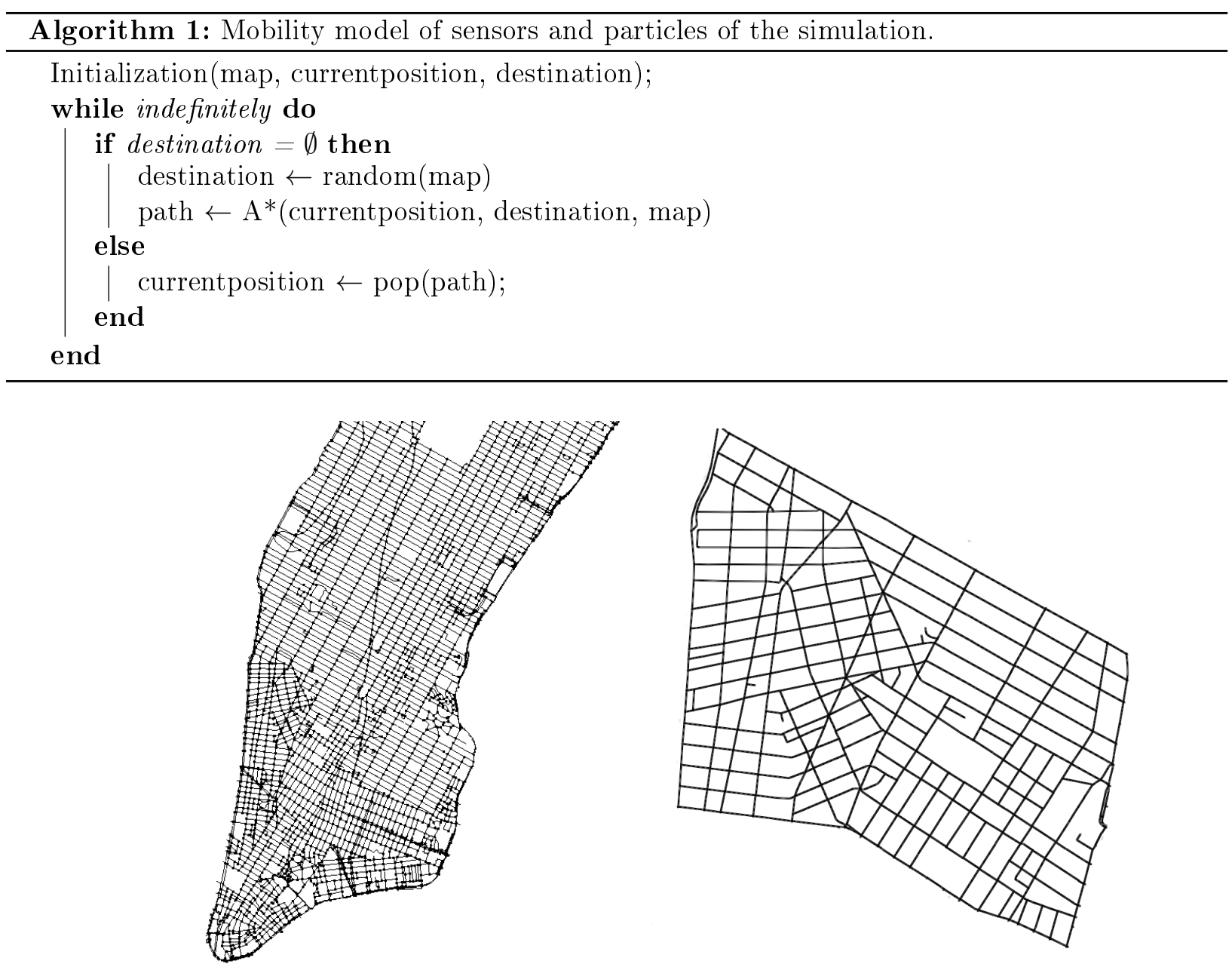

Figure 5.5: Maps used in the simulation. Edges represent navigable paths.

metric in Equation 5.8.

\subsection{Experimental Results}

This section is divided into three parts: person detection and density map generation; calibration of the simulation; and simulation. The source code is available at ${ }^{3}$.

\subsubsection{Person Detection Results}

We manually tagged 600 images to use as a ground truth. The R-FCN algorithm [25] was trained on the Pascal VOC 2007 dataset with the 101-layers neural network architecture proposed by [41]. We evaluated the method with detection scores ranging from 0.0 to 1.0, spaced by 0.1 .

None of evaluations achieved recalls exceeding $80 \%$ (see Figure 5.6) and this fact is inherent to the difficulty of object detectors in detecting small objects as discussed in Section 5.2.1. To mitigate such issue, the detection model we propose assumes a finite radius of coverage (see Figure 5.4) and thus, we establish a limit on the size of the objects detected in the image. Figure 5.6 shows the results of the adopted detector over our sample as we vary the minimum acceptable height. As we can see, the higher the ground-truth height threshold, the higher the precision and specially the recall of the method.

We utilized as our detector the R-FCN method [25] with a residual network of 101 layers architecture [41] trained on Pascal VOC 2007 [30]. The model was trained using a weight decay of

\footnotetext{
${ }^{3}$ https://github.com/VIDA-NYU/pedestrian-sensing-model
} 

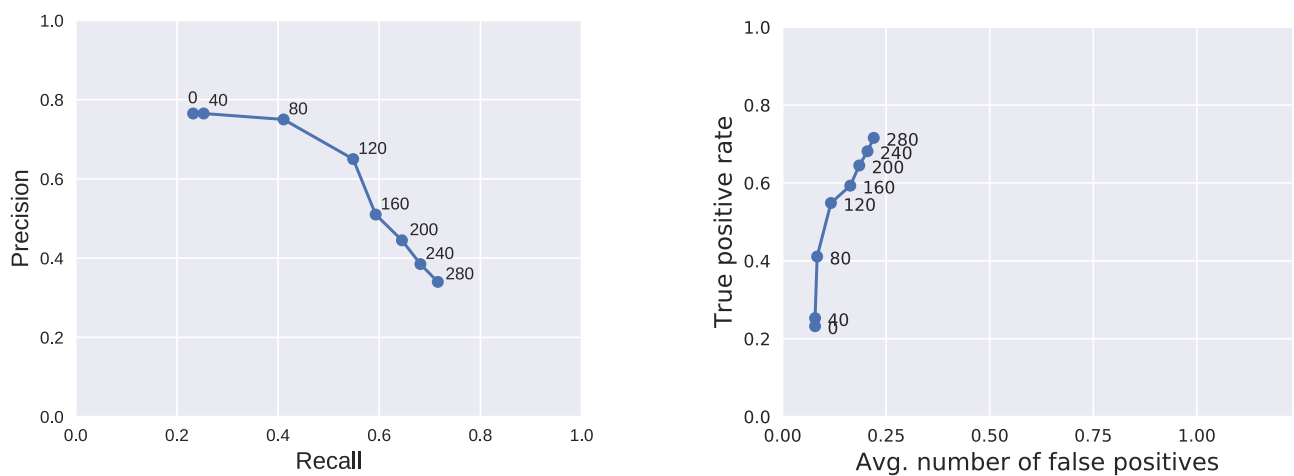

Figure 5.6: Evaluation of R-FCN [25] for different ground-truth height thresholds. The utilized model has a Resnet-101 backbone [41] trained on the Pascal VOC 2007 dataset [30].

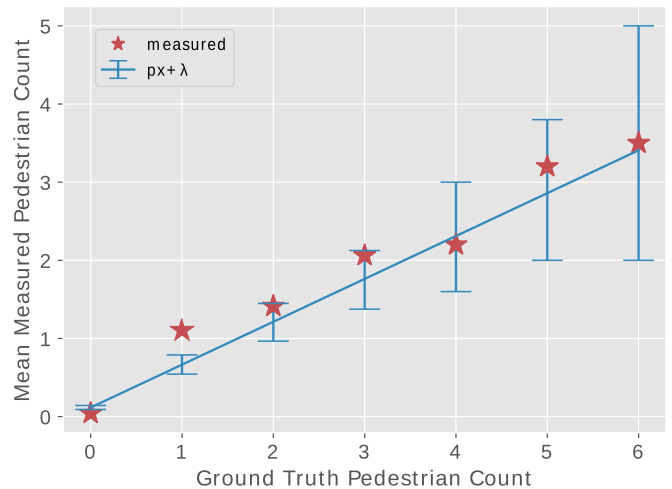

Figure 5.7: A comparison of the ground truth and the measured person count from the 600 tagged test images. While the actual true positive and false positive counts do not match the expected statistics (left), the total measured person count can be close to approximated as linear (right). It should be noted that this is only an approximation as, even taking sampling errors into account, the mean measured count do not fit a linear model. Error bars are the $95 \%$ confidence interval of the mean, calculated by assuming the sampling process described in Section 5.2.3. 


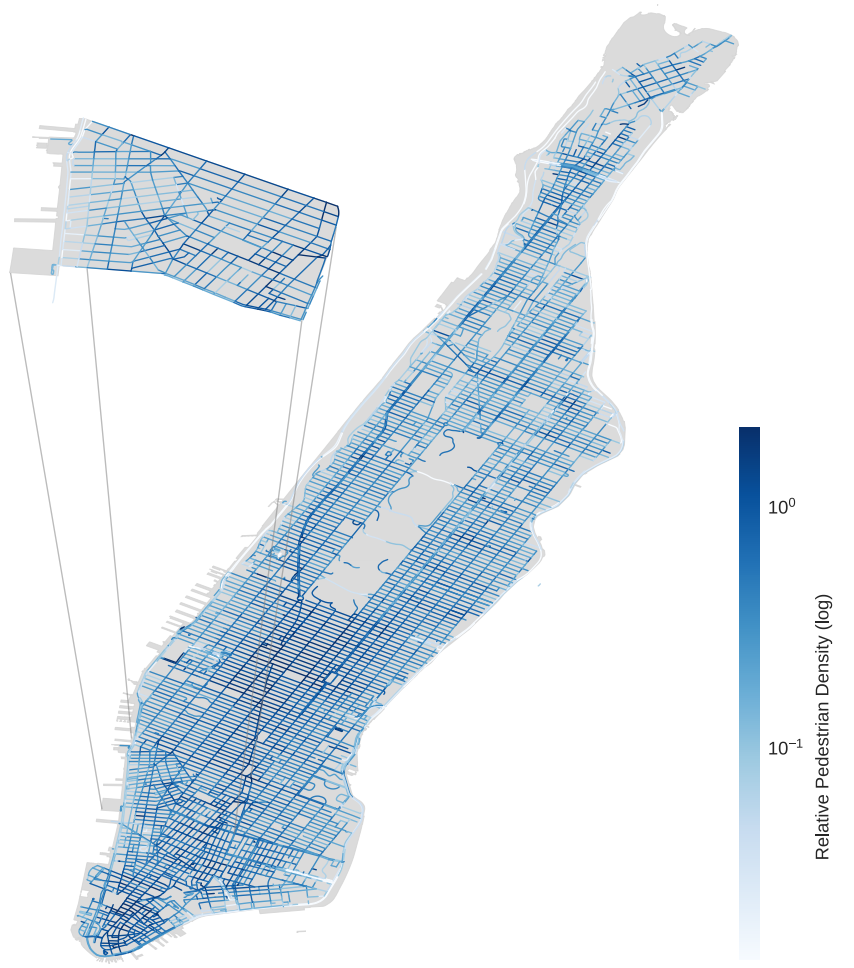

Figure 5.8: Visualization of the density of people in Manhattan. The scale of colors represent the relative density of people. The main figure depicts the heatmap over the island of Manhattan. The same heatmap is enlarged in the top-left of the figure to show the details of midtown and surrounding areas. Underlying map data taken from OpenStreetMap [88]. Not drawn to scale.

0.0005 and a momentum of 0.9 . Assuming a method minimum score of 0.7 and height threshold of 120 pixels, overall 7,474,623 people were detected.

As shown in Figure 5.7, the total number of people detected is approximately linear satisfying the requirements of the model proposed in Section 5.2.3, despite stastically significant deviations. These stem from the visions algorithm's better than expected performance for images without any person and worse than expected performance for images with a single person. While we do not know how these deviations would effect the error bounds given in Equations 5.9 and 5.10, we hypothesize that the two deviations would cancel themselves out and bound may still approximately hold with a slightly larger equivalent $\lambda$.

A visualization of the density of people in entire Manhattan can be seen in Figure 5.8. For each analyzed picture, the number of people is determined using the detection algorithm and these counts are aggregated by streets. The topology of the streets were obtained from OpenStreetMaps [88]. The samples were organized in a KD-tree [13] and aggregated by street segments. To account for the imbalance of the number of images per segment, the average of the counts is considered.

Distribution of the number of people, like ours, can be useful for city planing, commercial, and other purposes. Taxis seeking riders, food trucks seeking customers, and businesses seeking storefronts all benefit from large crowds. However, traffic and self-driving cars do not. A knowledge of the distributions of the number of people can allow city planers, civil engineers, and traffic engineers to make better decisions.

Our person map can also show the effect that features of the city have on it's people. In addition to populated neighborhoods, subway stations, and attractions like the Metropolitan Museum of Art are all associated with a spike in the person densities. These spikes might be too localized to be detected using traditional methods. Further studies of vision based person counts may lead to a 
better understanding of the interplay between a city's environment and it's occupant's walking habits.

\section{Special Cases}

A visual inspection of the detections showed that the methods are scale-sensitive, having difficulty to detect tiny-scale objects. However, when the objects have representative sizes, even in crowded scenes, the method proved to be robust. Another difficulty of the method regards the differentiation between pedestrians and non-pedestrians, such as cyclists and disabled people (see Figure 5.9). Such differentiation would require a novel computer vision method that considered the context of the scene. We performed a manual inspection over our annotated dataset and cyclists represented $2.4 \%$ of all annotated pedestrians. This low rate supports the argument that such limitation of the method does not invalidate the results statistically.
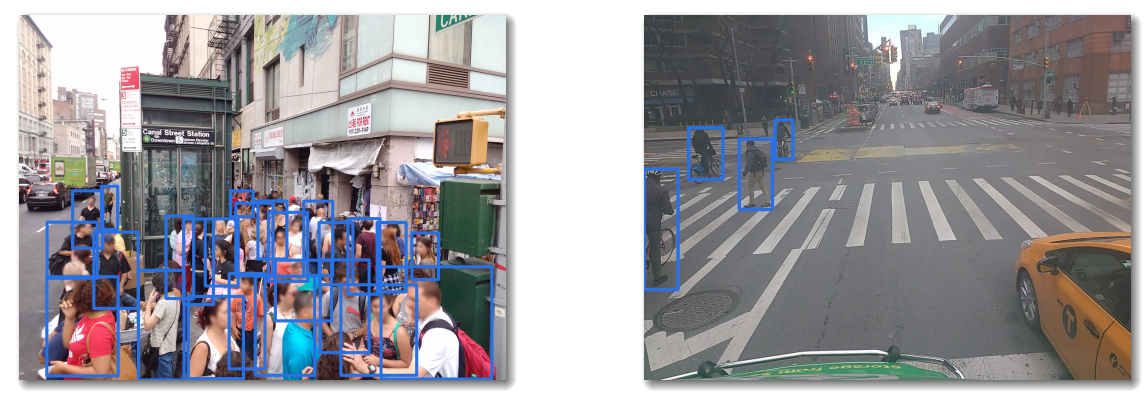

Figure 5.9: Person detection by the method in special cases. On the left, person detection in a crowded scene. On the right, pedestrians and non-pedestrians (a cyclist) are equally identified by the method.

\subsubsection{Simulation Calibration}

Simulation parameters have been calibrated according to the computer vision experiments. In Figure 5.10 we can see in red the expected number of false positives and the true positive rate of the computer vision method utilized. Given the average people density $(\hat{h})$ of 0.587 obtained in the previous section, following Equation 5.9, an error of 0.062 is obtained. The actual error may be larger due to the deviations from linearity previously discussed.

We compared the number of measured person counts as a function of the average number of ground truth people in each of the 600 manually labeled images to test the linear assumption used in Equation 5.5. Error bars for the mean were computed using the 5\% to $95 \%$ values of the median

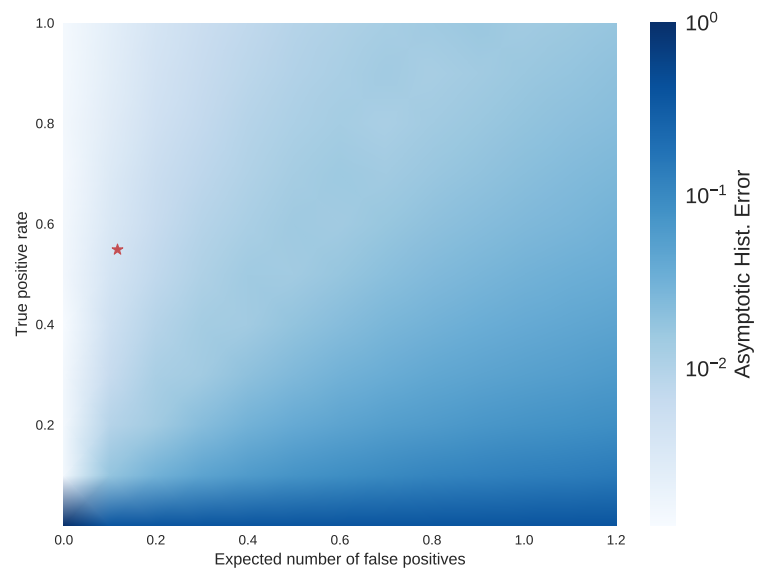

Figure 5.10: Asymptotic error metric between the sensed and ground truth histograms in our simulation. Plotted as a function of the sensors true positive and expected number of false positives. 
of the appropriate sample process given in section 5.2.3. We measured the true positive rate $(p)$ to be 0.54 and the average number of false positives $(\lambda)$ to be of 0.117 . 


\subsubsection{Simulation Results}

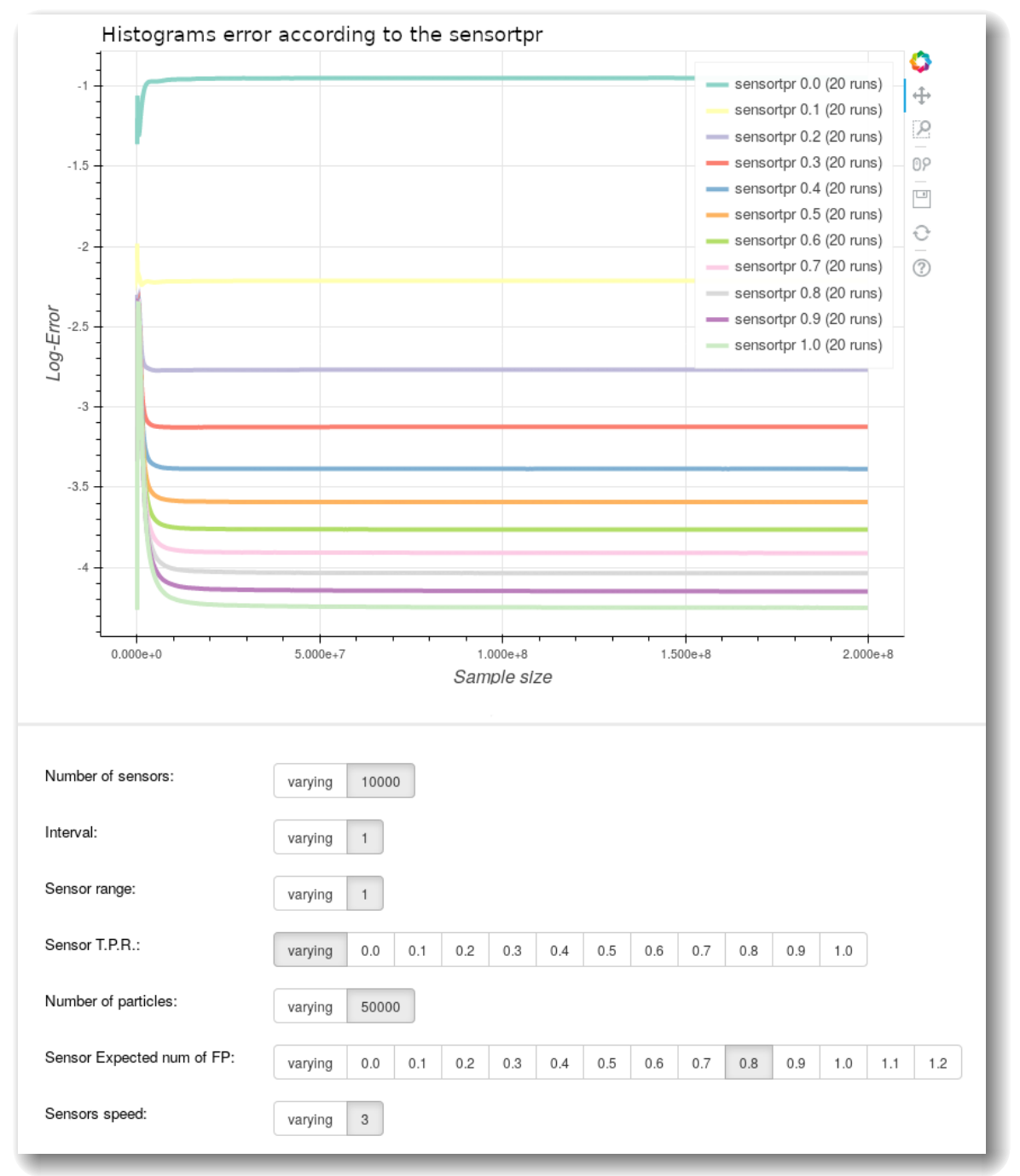

Figure 5.11: Interface created to visually explore the results of the simulation.

We evaluated different true positive rates and expected number of false positives of the sensors, $p$ and $\lambda$ and we used a Mersenne Twister pseudo number generator [78]. The various values for the parameters used in our experiments are listed in Table 5.2. For the 143 possibilities combination of values, we ran the simulation for 20,000 time steps 20 independent times.

The code is primarily implemented in Python with performance-sensitive sections implemented in Cython [12]. The average time to run a single experiment of this optimized code is of 11,718 seconds. Running the experiments on a single machine would take roughly one year of computation. By running them in parallel, it took 11 days.

For each experiment we examine the decay of the metric given by Equation 5.8 as a function of the cumulative number of samples captured by all the sensors. We assume the error continues to decay until it reaches an asymptotic minimum error within the 20,000 simulation time steps. Afterwards, we take the average decay curve of all 20 runs for each settings configuration and take the average of the last 200 values to find the asymptotic value.

We can visualize the results from the simulations in Figure 5.12 which shows how the variation of true positive rate and false positive rate affect our histogram error. If we take a horizontal profile of say 0.2 of true positive rate we can see how the errors are greatly affected by the variation of the expected number of false positives, varying from very low to high error values (represented by the variation on the color saturation). We compare these values to our theoretical formulas (see Equation 5.9), and show they are approximately equal. Finally, We show that they are within the 
Table 5.2: Parameters of the simulation along with the values we used in the experiments.

\begin{tabular}{|c|c|c|}
\hline Parameter & Symbol & Values \\
\hline Number of people & $N_{\text {people }}$ & 50000 \\
\hline Person speed & $\nu_{\text {person }}$ & 1 \\
\hline Sensor speed & $\nu_{\text {sensor }}$ & 3 \\
\hline Number of sensors & $N_{\text {sensors }}$ & 10000 \\
\hline Sensor true positive rate & $p$ & $\{0.0,0.1,0.2,0.3,0.4,0.5,0.6,0.7,0.8,0.9,1.0\}$ \\
\hline Sensor exp. number of false positives & $\lambda$ & $\{0.0,0.1,0.2,0.3,0.4,0.5,0.6,0.7,0.8,0.9,1.0,1.1,1.2\}$ \\
\hline Sensor range & $r$ & 1 \\
\hline
\end{tabular}

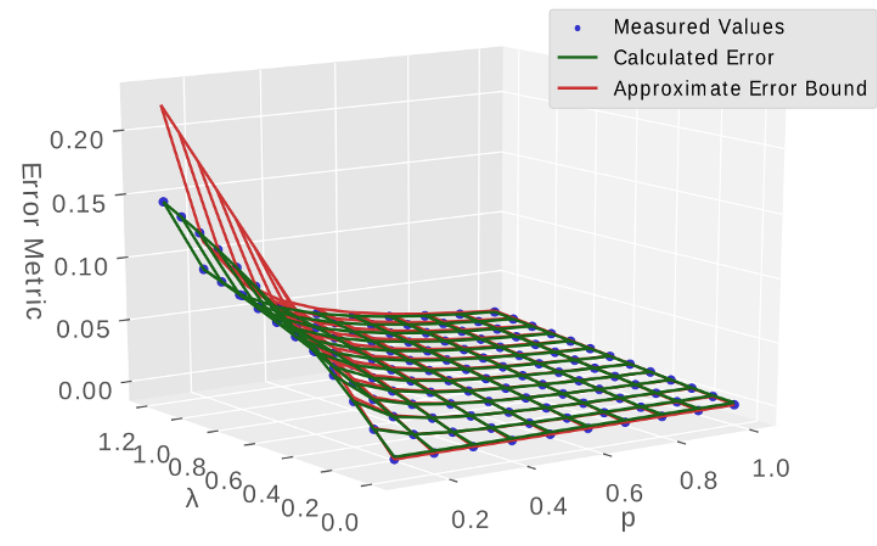

Figure 5.12: Comparison of the asymptotic histograms error measured from the simulation experiments (blue dots) to the theoretical close form function and the approximate bound (please refer to Equation 5.9, red mesh). 
bound given by Equation 5.9. 


\subsection{Conclusion}

In this chapter we applied computer vision techniques over a large and novel set of streetlevel images to obtain a pedestrian density map of the region of Manhattan. Inspired by the density estimation procedure we proposed a sensing model and established theoretical bounds for the density estimation error. Results give evidence that even when considering faulty sensors such as abstracted by our detection algorithms, reliable density maps can be obtained under our model assumptions.

Besides the results presented, there are other potential future avenues of studies as discussed next. First, we should caution that any application of our methodology should perform statistical tests to ensure that their results are statistically significant. While we set bounds on the asymptotic error after the sampling process converges, we have only provided case studies and heuristics for the time to convergence. It would be interesting to find a formal bound on time to convergence as well as provide guidelines for the appropriate statistical tests to validate the data post collection.

Our experiments could be extended to consider alternative mobility models [16], as well as dynamics models including macroscopic ones [42, 43, 47]. We can also use data completion algorithms $[34,63,65]$ to reconstruct a city-wide people map.

The people distribution generated will then be able to be combined with other urban datasets such from Socrata [86], weather, crime rate, vandalism [120], census data, public transportation, bicycles and shadows [80]. We additionally aim to explore apparently disparate datasets such as from wind and from garbage collection.

Another future work is incorporation of advances such as from [92] to visualize our images in the context of the city and use this visualization to gain additional insights into other datasets.

Additionally, we hope to use 3D pop-ups and/or photo based rendering to fully enhance the images in the three dimensional environments. 


\section{Chapter 6}

\section{Graffiti Distribution from Ground-Level Images}

This chapter covers the application of graffiti quantification in cities by usage of ground-level images and it is an adapted version of [120] in which this chapter is based on ${ }^{1}$.

\subsection{Introduction}

Graffiti is already part of the landscape of most megacities. It can be categorized as artistic drawings or tagging (see Figure 6.1) and whereas graffiti drawings require talent and practice, most of the times graffiti tagging represents an unauthorized act that people convey messages or show their names. The discussion whether graffiti drawing is an art is extensively debated [79, 142]. In 2017 a Brooklyn based company was fined 6.7 million dollars for whitewashing the murals containing famous graffiti ${ }^{2}$. This debate is less controversial when considering the latter kind of graffiti and public authories repudiate and combat it. In this chapter, focus is given to this latter type and they are referred as simply graffiti in this work.
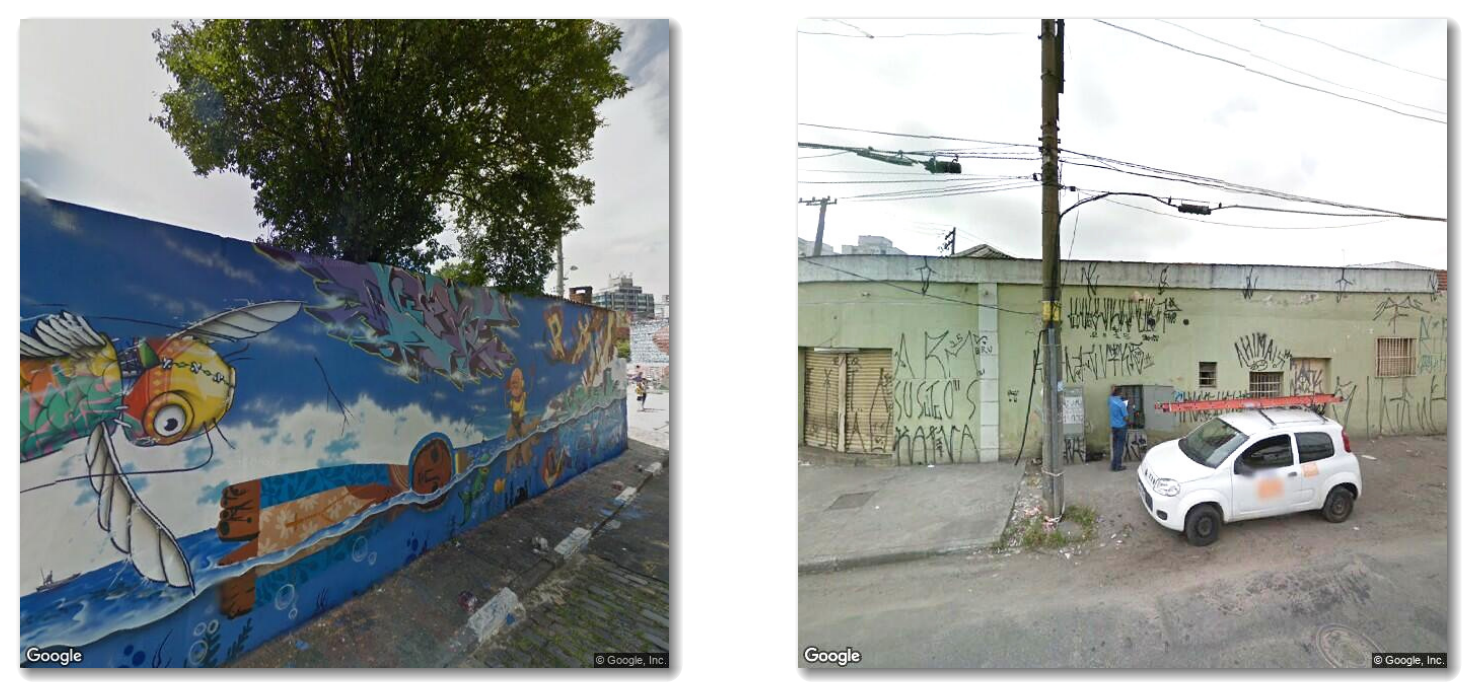

Figure 6.1: Different types of graffiti. The wall pictured on the left contains a detailed and artistic drawing and it is reasonable to suppose that skill and time were necessary to paint it whereas the graffiti on the right is composed of words and symbols. In this work, there is special interest in the graffiti type on the right. Images from [38].

There are several works aimed to combat graffiti vandalism acts. Some web-based frameworks [8, $73,6,131]$ rely on community participation to identify recently degraded locations while some works

\footnotetext{
${ }^{1}$ This work has been carried out in a collaboration with professors Roberto M. Cesar-Junior and Claudio T. Silva.

${ }^{2}$ https://www.nytimes.com/2018/02/12/nyregion/5pointz-graffiti-judgment.html
} 
$[4,28,124]$ try to identify the real drawing act. Other works retrieve similar graffitis in a reference database [139] by using connected components and keypoint matching in an attempt to associate graffiti with gangs. Alternatively, [125] attempt to identify the graffiti authorship. Given a target image, they compute a metric based on the symbols contained, manually annotate and do a keypoint matching between the images and the gang graffitis.

Semantic segmentation is a high-level computer vision task which aims to split the image into known classes [5]. It is a complex task when compared to image classification and object detection because it requires the image pixel-wise classification. Research on semantic segmentation is very active and recent works achieve impressive results [5, 101, 9, 148, 74]. A related task is the instance segmentation, where the objective is also to identify the instances but in contrast to the ordinary segmentation, the method must be able to identify the boundaries of two adjacent instances. Some previous works $[93,24]$ performed this task by preceding the object detection stage with a segmentation stage. The work of Mask-RCNN [40] in turn performed this task by running the classification and the segment proposals in a parallel manner. [40] relies on FRCNN [100] architecture but with an additional branch for instance segmentation.

Google Maps [38] provides public access to images captured by cars driven down the streets. Images are obtained from different geographical locations and in different views and formats. Many works [103, 126] have already utilized this type of imagery for urban analyzes. The authors of [29] use street view images to compare the architectural elements from different cities. In [103], a study on the feasibility on audits of neighbourhoods environments instead of in-person auditing is presented. The work [66] proposes the assessment of urban greenery using the same kind of images.

There is no graffiti map of the São Paulo city and creating it by manual inspection would demand great effort. In this work, the creation of a graffiti map based on the segmentation of graffiti regions on street view images [38] is proposed.

Question 5: Can ground-level images obtained from a wireless sensors network be used to compute the graffiti map of a city?

The contribution described below is application-oriented and can be summarized as the proposal of an automated way to quantify the level of graffiti in a location given a pool of ground-level images from the location. For this task, ground-level images are systematically acquired, computer vision algorithms identify and quantify the amount of graffiti in a picture and finally a new metric, the graffiti level of the region is devised and computed. A case study was performed in a highly urbanized city, São Paulo, Brazil

\subsection{Proposed method}

In order to confidently estimate the level of graffiti in a geographical region, a metric, the graffiti level, obtained through the identification and computation of the areas containing graffiti on street view imagery [38] is introduced.

\section{Acquisition}

The region of interest is initially defined and the images are acquired. Due to limitations on the coverage of the pictures provided and on computational constraints, a sample of the full region is considered. There are a number of ways of performing sampling [113] but they can be classified as random sampling methods or systematic sampling methods. The first removes the bias of the selection by randomly selecting the sample points although not guaranteeing good coverage. The latter, in contrast, assures coverage by including bias.

Once the geographical sample is defined, ideally a full view should be considered for each geographical location. A single $360^{\circ}$ panorama view can be used, but one may need to worry about the distortions present in panoramic photos. Alternatively, complementary views for each location may be considered (see Figure 6.2). 

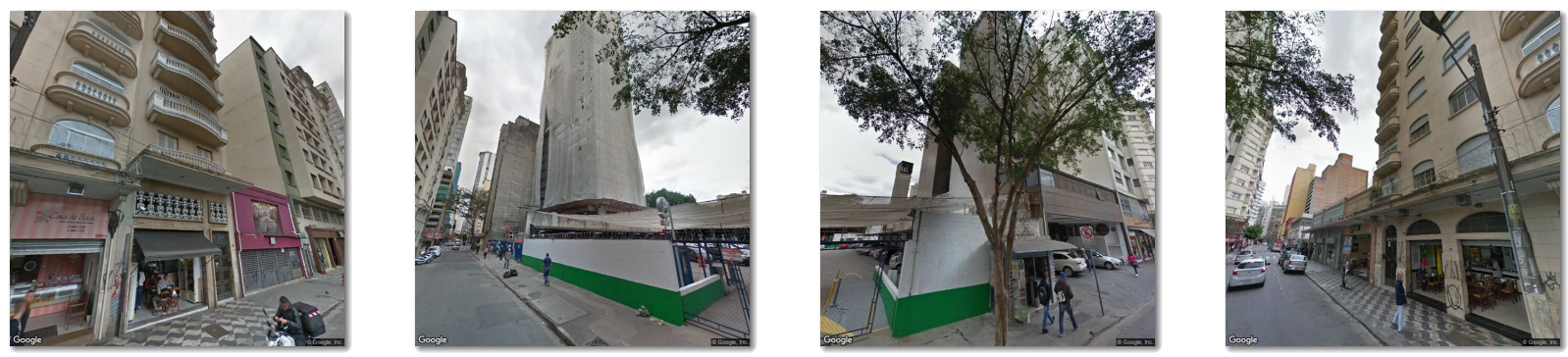

Figure 6.2: Four views from the same geographical location. Images obtained from [38].

\section{Graffiti recognition}

Given the objective of quantifying the level of graffiti in a given location, a simple and direct way would be to identify the images containing or not graffiti. Such characterization however give us a sharp and non-precise information of each picture. It would be interesting to have a more granular value for each picture. So the graffiti level $f(P)$ of a geographical location $P$ is defined as the total area $A$ of the picture containing graffiti. Such approach can be affected by the projection map of the scene and also by the distance of the camera to the region containing graffiti. Different regions, given a minimum extent, are assumed to have corresponding distribution of projections and of distance to the walls and with this assumption, $f(P)$ may be used to compare different geographical locations. Since each location $P$ is represented by a set of $k$ views, $f(P)$ is defined as the sum of the areas of the regions containing graffiti in each view (see (6.1)). The graffiti level of a region $(g(R))$ can then be aggregated by geographical regions by computing the average of the graffiti levels on the obtained sample of size $n$ (see Equation (6.2)) .

$$
\begin{gathered}
f(P)=\sum_{i=1}^{k} A_{i} \\
g(R)=\frac{\sum_{j=1}^{n} f\left(P_{j}\right)}{n}
\end{gathered}
$$

The Mask-RCNN [40] method was chosen for the segmentation task given its high performance reported on important benchmarks [22, 70]. During training, the method minimizes a multi-task loss $L=L_{c l s}+L_{b o x}+L_{\text {mask }}$, being the classification loss $L_{c l s}$ and bounding-box loss $L_{b o x}$ the same as defined in [100] and the mask loss $L_{\text {mask }}$ defined as the average binary cross-entropy loss. Despite the method segmentation outputs and instance information, this work just uses the segmentation results.

Since there is no dataset publicly available, a dataset with manually annotated images was created and used to train the model.

\subsection{Experiments}

Initially a pilot sample of 10,000 street view images from [38] from São Paulo City was collected and a sample was manually chosen. The regions containing graffiti tags were manually identified. A total 632 of images were manually annotated and used to train Resnet 101-layers backbone [41] pre-trained on the COCO dataset [70]. A learning rate of 0.001 and a momentum of 0.9 was adopted and the model was trained for 80 epochs. The utilized model was from epoch 30, given its highest validation error (see Figure 6.3). The final model showed an average precision [30] of 0.57. Figure 6.5 presents a sample of the detections evaluated. The full time to process a single image is of $0.69 \mathrm{~s}$ on a Geforce GTX 1050.

In Figure 6.4 the heterogeneous coverage of the service utilized [38] in the city can be seen. The two bottommost districts showed little coverage at the time of the acquisition, given the 


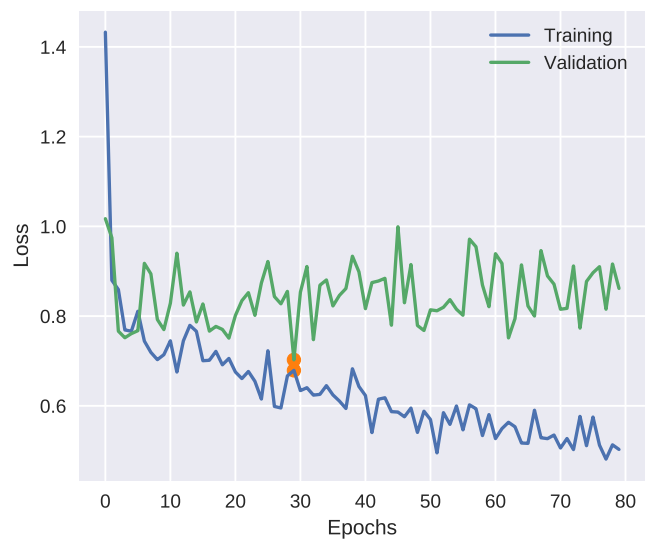

Figure 6.3: Training and validation loss of the [40]-based model during the training stage. The highlighted points (epoch 30) correspond to the final model training and validation accuracy.

predominantly rural and unpopulated nature of these regions and thus they were not considered in this study.

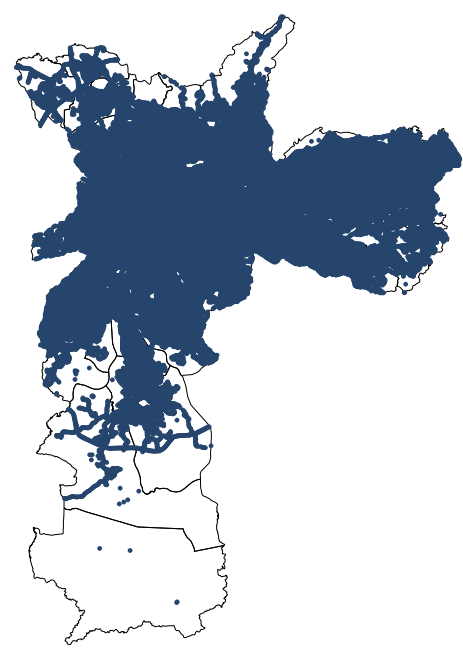

Figure 6.4: Coverage of the utilized images using [38] images. Dots represents existence of pictures in the region. Low coverage can be observed in the bottom of the map.

Four views for each geographical location were utilized, spaced by $90^{\circ}$. Notice in Figure 6.2 how the scene elements from the second and the third figures intersect which indicates a full coverage for each geographical location. The majority of the images considered are from 2017 as can be seen in Table 6.1.

Table 6.1: Acquisition year of the geographical location analyzed. Metadata provided by [38].

\begin{tabular}{lccccccccc}
\hline Year & 2010 & 2011 & 2012 & 2013 & 2014 & 2015 & 2016 & 2017 & 2018 \\
\hline Points & 1,241 & 16,311 & 207 & 422 & 2,182 & 4,563 & 4,211 & 39,391 & 317 \\
\hline
\end{tabular}

A grid over the spatial extent of the city with 134,624 points was created with $102 \mathrm{~m}$ vertical and horizontal spacing. After eliminating images from third-party providers and non-mapped regions (see Figure 6.4), a geographical coverage of 68,752 geographical points and 275,339 images overall was obtained.

In Figure $6.6 \mathrm{~b}$ it can be seen that except a small region inside the map, the regions with highest 

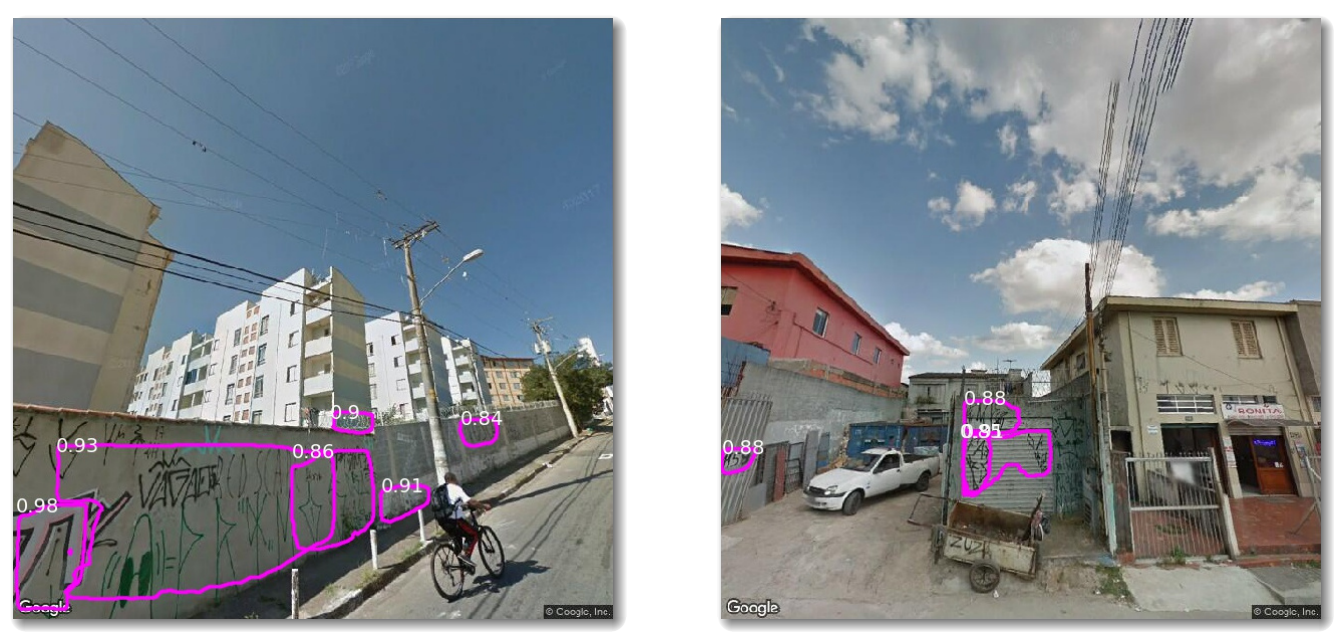

Figure 6.5: Sample of the graffiti detections. The values depicted represent the confidence of the detections.

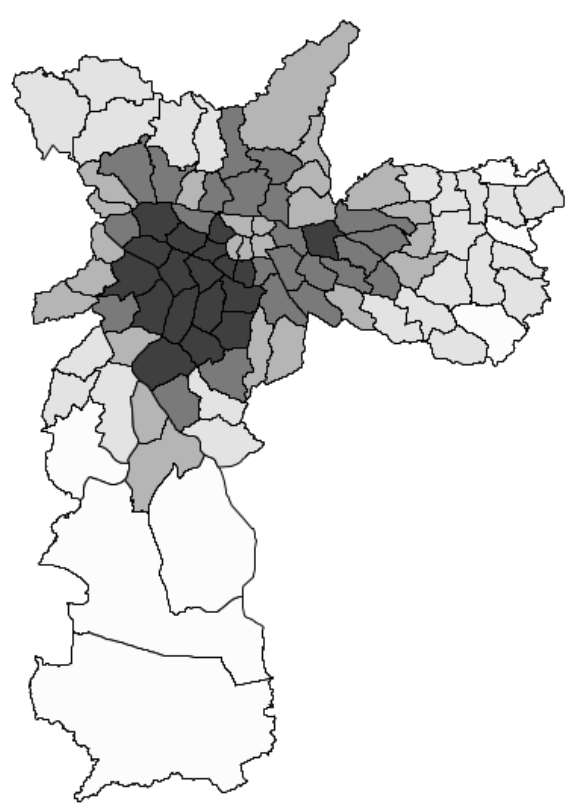

(a) $H D I$

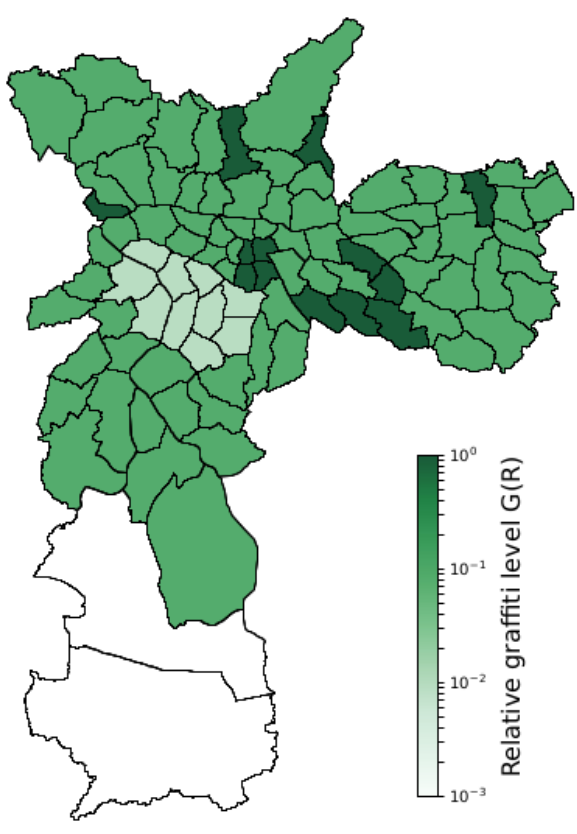

(b) Graffiti map

Figure 6.6: (Left) Human development Index from São Paulo districts in 2007 [117]. (Right) Relative graffiti level in São Paulo city. The divisions represent the city districts and the colors in logarithmic scale express the relative graffiti level, according to Equation 6.2. Bottommost regions were not considered given the unavailability of data in the region.

levels of graffiti are in peripheral regions of the city. The regions with lowest levels of graffiti are in the business center of the city. The bottommost parts were not considered given the coverage of the service utilized.

The Human Development Index (HDI) is a development measure of a region that considers life span, income and education aspects [45]. Figure 6.6a is a HDI heat-map by districts using the data released by the city hall [117] in 2007. Notice that the regions with the lowest levels of graffiti in Figure $6.6 \mathrm{~b}$ correspond to the regions of highest HDIs in Figure 6.6a. 


\subsection{Conclusions}

This chapter describes a work on automatic mapping of regions containing graffiti tags. Ground level imagery was systematically obtained from [38] and the graffiti tags in each image were identified and a metric for the graffiti level of a geographical region was finally proposed. A case study in São Paulo was conducted and it was shown that it is in accordance to what is expected given the indicators of HDI.

There are limitations of the proposed approach. One of them is the requirement of sampling, due to computational constraints. Small regions with highly concentrated tagging do not properly contribute to the metric of the region. Future steps include the utilization and the combination of vision algorithms [69] with higher performances and the use of semi-supervised approaches to overcome the lack of annotated data. Additionally, one may consider a denser sampling, a joint analysis with other geographical regions and the use of new datasets that include the same view in different times. 


\section{Chapter 7}

\section{Conclusions}

\section{Concluding Remarks}

Thanks to advances in a number of fields, technology is quickly advancing. Data is generated in high speed and large volumes. Visual data generation is performed in diverse forms and scales but centralized ways of generating ground-level imagery includes surveillance camera network and fleets of camera-equipped vehicles. The data generated in such ways has incommensurable value, and in this work challenges and applications on the usage of this kind of data was explored. Initially, in Chapter 3 the object detection methods evaluation in a scarce and expensive annotations scenario is studied and a model with cheaper despite less detailed computation is proposed. In Chapter 4, the impact of the rain to the object detection task using low resolution surveillance images is studied and a strong indication of the inefficiency of it has been obtained. In Chapter 5, a model that takes into account the uncertainty of real sensors is proposed and bounds are estimated, showing that the sensors inefficiency may impact the validity of the final results. These results are utilized to compute a pedestrian distribution map using state-of-the-art CV methods from a large set of images obtained from a mobile sensor network. In Chapter 6 , a similar approach was applied to the problem of graffiti distribution, with proper adaptations according to the object of interest and to the images source. From these findings, it is clear that in fact there is great value in ground-level images when coupled with $\mathrm{CV}$ techniques, but it comes with challenges associated.

\section{Future Work}

There are many ways to extend this work. In Chapter 3, alternative semi-supervised strategies as well as active learning approaches could be explored. Extensions to Chapter 4 include the development of task-based quality assessment for de-raining methods and more complex rain formation models, that combine different types of rain and also reinforcement learning approaches can be adapted to gradually de-rain the target image. In Chapter 5, heterogeneous and more complex mobility models can be used for the agents in the simulation. In Chapter 6 , different models can be tested to improve the accuracy of the segmentation. Also, it can be extended to different cities in the world.

\section{List of publications}

The results of this thesis resulted in the publication in three conferences until the moment:

(i) Tokuda, E.K., Ferreira, G., Silva, C.T. and Cesar-Jr., R.M.. A novel semi-supervised detection approach with weak annotation. In IEEE Southwest Symposium on Image Analysis and Interpretation (SSIAI), pp. 129-132. IEEE, 2018. 
(ii) Tokuda, E.K., Silva, C.T., and Cesar-Jr., R.M.. Identificação automática de pichação a partir de imagens urbanas. In Conference on Graphics, Patterns and Images (SIBGRAPI) Workshop. 2018 .

(iii) Tokuda, E.K., Silva, C.T., and Cesar-Jr., R.M.. Quantifying the presence of graffiti in urban environments. In Big Data and Smart Computing (BigComp), 2019 IEEE International Conference on. IEEE. 2019.

(iv) Li, Siyuan, Araujo, I., Ren, W., Wang, Z., Tokuda E. K., Hirata-Jr., R., Cesar-Jr., R., Zhang, J., Guo, X., Cao, X., 2019. Single Image Deraining: A Comprehensive Benchmark Analysis. In IEEE Conference on Computer Vision and Pattern Recognition (CVPR), USA, 2019.

(v) (To appear). Tokuda, E.K., Lockerman, Y., Ferreira, G., Sorrelgreen, E., Boyle, D., Cesar-Jr., R.M. and Silva, C.T.. A new approach for pedestrian density estimation using moving sensors and computer vision. Accepted in ACM-TSAS.

Works (iii, v) resulted from the collaboration with NYU and work (iv) from the collaboration with professor Zhangyang Wang from Texas A\&M University. 


\section{Acknowledgements}

The author thanks CAPES and São Paulo Research Foundation (FAPESP) grants numbers 2014/24918-0,2016/12077-6,2015/22308-2 for the financial support because without it, none of this would have been possible.

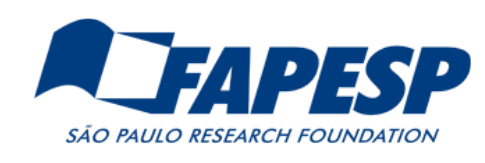

The author recognizes the importance of FAPESP support to the project.

Special thanks to the collaborators, including C. T. Silva, for the warm reception in New York city; Z. Wang, for the prolific technical discussions; Carmera, for providing the data and being always solicitous. 
CONCLUSIONS 


\section{Appendix A}

\section{Proof of Metric Formula}

Here we derive the formula for the closest point in our class and the ground truth vector. This is equivalent to solving:

$$
\min _{a}|a \psi-\phi|^{2}
$$

First we expand the distance metric using the euclidean inner-product:

$$
\begin{aligned}
|a \psi-\phi|^{2} & =<a \psi-\phi, a \psi-\phi> \\
& =a^{2}<\psi, \psi>-2 a<\psi, \phi>+<\phi, \phi>
\end{aligned}
$$

which is minimized by

$$
a=\frac{<\psi, \phi>}{<\psi, \psi>}=\frac{<\psi, \phi>}{|\psi|^{2}}
$$

when $|\psi|^{2} \neq 0$. For $|\psi|^{2}=0$, then $\psi=0$ and

$$
\min _{a}|a \psi-\phi|^{2}=|\phi|^{2}
$$

regardless the value of a.

Substituting this value into $a$, we obtain our equation above:

$$
\psi^{\prime}= \begin{cases}\psi \frac{\leq \psi, \phi>}{|\psi|^{2}} & |\phi| \neq 0 \\ 0 & |\phi|=0\end{cases}
$$

When $\langle\psi, \phi\rangle=0$ then $\psi^{\prime}=0$ and we get the same value.

\section{A.1 Theoretical Asymptotic Error}

In this paper we will derive the equation for sensor error that we give in Equation 5.9. The error bounds only assume that $E[\psi(x)]=p \phi(x)+\lambda$ for some values of $p$ and $\lambda$. First, we will show that, for the simulation, the values of $p$ and $\lambda$ agree with the parameters of the same name.

As noted in Equation 5.3, the sampling process for the simulation results in the following sampled values for each location:

$$
\psi(x)=\frac{1}{k} \sum(T(n(x))+F)
$$

where $T(n(x))$ is a sampled from a binomial distribution with mean $n(x)$ and $F$ is a Poisson process [52] with a mean of $\lambda$. 
At the same time, the ground truth distribution of people at each location is given by Equation 5.4

$$
\phi(x)=\frac{1}{s} \sum n(x)
$$

In this appendix we will make the simplifying assumption that $s=k$

The executed value of the sampled can then be found by (noting that the random variables are all independent)

$$
\begin{aligned}
E[\psi(x)] & =\frac{1}{s} \sum(E[T(n(x))]+E[F]) \\
& =\frac{1}{s} \sum(p n(x)+\lambda) \\
& =p \frac{1}{s} \sum(n(x))+\lambda \\
& =p \phi(x)+\lambda
\end{aligned}
$$

From this point on, all results will only depend on the equations $E[\psi(x)]=p \phi(x)+\lambda$ and not the underlying sampling process.

Let $m$ be the total number of people and $r$ be the sampling location. In a real world scenario, $m$ may not be well defined. As such, we will work in terms of $h=\frac{m}{r}$, the density of people.

We want to calculate $\frac{\left|\psi^{\prime}-\phi\right|}{\left|\psi^{\prime}\right|+|\phi|}$ and we are going to compute each term separately.

By the law of large numbers, in the limit of $s \rightarrow \infty, \psi$ approaches

$$
\psi(x) \rightarrow E[\psi(x)]=\phi(x) p+\lambda
$$

Using this limit, we can find the asymptotic value of $\psi^{\prime}$, as defined by Equation 5.7, can be found:

$$
\begin{aligned}
\psi^{\prime} & =\psi \frac{\langle\psi, \phi\rangle}{|\psi|^{2}} \\
& \rightarrow(p \phi+\lambda) \frac{\langle p \phi+\lambda, \phi\rangle}{|p \phi+\lambda|^{2}} \\
& =(p \phi+\lambda) \frac{p|\phi|^{2}+\lambda\langle 1, \phi\rangle}{p^{2}|\phi|^{2}+2 p \lambda\langle 1, \phi\rangle+\lambda^{2}\langle 1,1\rangle} \\
& =(p \phi+\lambda) \frac{p|\phi|^{2}+m \lambda}{p^{2}|\phi|^{2}+2 m p \lambda+r \lambda^{2}} \\
& =\left(\phi+\frac{\lambda}{p}\right) \frac{p^{2}|\phi|^{2}+m p \lambda}{p^{2}|\phi|^{2}+2 m p \lambda+r \lambda^{2}}
\end{aligned}
$$

Here, $\langle\cdot, \cdot\rangle$ is the Euclidean inner product and 1 is the vector with all ones. Note that $\langle 1, \phi\rangle=m$ and $\langle 1,1\rangle=r$

The magnitude of $\psi^{\prime}$ can then be found by

$$
\begin{aligned}
\left|\psi^{\prime}\right| & =\frac{p^{2}|\phi|^{2}+m p \lambda}{p^{2}|\phi|^{2}+2 m p \lambda+r \lambda^{2}} \sqrt{\left.\left\langle\phi, \frac{\lambda}{p}\right\rangle\right\rangle} \\
& =\frac{p^{2}|\phi|^{2}+m p \lambda}{p^{2}|\phi|^{2}+2 m p \lambda+r \lambda^{2}} \sqrt{|\phi|^{2}+\frac{r \lambda^{2}}{p^{2}}+2 \frac{m \lambda}{p}} \\
& =\frac{p|\phi|^{2}+m \lambda}{p^{2}|\phi|^{2}+2 m p \lambda+r \lambda^{2}} \sqrt{p^{2}|\phi|^{2}+r \lambda^{2}+2 m p \lambda}
\end{aligned}
$$


Similarly, the difference between $\psi^{\prime}$ and the ground truth sampling can be found by

$$
\begin{aligned}
\psi^{\prime}-\phi & =\left(\phi+\frac{\lambda}{p}\right) \frac{p^{2}|\phi|^{2}+m p \lambda}{p^{2}|\phi|^{2}+2 m p \lambda+r \lambda^{2}}-\phi \\
& =\left(\phi+\frac{\lambda}{p}\right) \frac{p^{2}|\phi|^{2}+m p \lambda}{p^{2}|\phi|^{2}+2 m p \lambda+r \lambda^{2}}-\phi \frac{p^{2}|\phi|^{2}+2 m p \lambda+r \lambda^{2}}{p^{2}|\phi|^{2}+2 m p \lambda+r \lambda^{2}} \\
& =\phi \frac{p^{2}|\phi|^{2}+m p \lambda-p^{2}|\phi|^{2}-2 m p \lambda-r \lambda^{2}}{p^{2}|\phi|^{2}+2 m p \lambda+r \lambda^{2}}+\frac{\lambda}{p} \frac{p^{2}|\phi|^{2}+m p \lambda}{p^{2}|\phi|^{2}+2 m p \lambda+r \lambda^{2}} \\
& =-\phi \frac{m p \lambda+r \lambda^{2}}{p^{2}|\phi|^{2}+2 m p \lambda+r \lambda^{2}}+\frac{\lambda}{p} \frac{p^{2}|\phi|^{2}+m p \lambda}{p^{2}|\phi|^{2}+2 m p \lambda+r \lambda^{2}} \\
& =\frac{\lambda}{p^{2}|\phi|^{2}+2 m p \lambda+r \lambda^{2}}\left(-\phi(m p+r \lambda)+p|\phi|^{2}+m \lambda\right)
\end{aligned}
$$

The magnitude of which can be found by

$$
\begin{aligned}
\left|\psi^{\prime}-\phi\right| & =\frac{\lambda}{p^{2}|\phi|^{2}+2 m p \lambda+r \lambda^{2}} \sqrt{\left\langle-\phi(m p+r \lambda),\left(p|\phi|^{2}+m \lambda\right) 1\right\rangle} \\
& =\frac{\lambda}{p^{2}|\phi|^{2}+2 m p \lambda+r \lambda^{2}} \sqrt{|\phi|^{2}(m p+r \lambda)^{2}+\left(p|\phi|^{2}+m \lambda\right)^{2} r-2(m p+r \lambda)\left(p|\phi|^{2}+m \lambda\right) m}
\end{aligned}
$$

Equations A.7 and A.9 can be used to find our metric as defined by Equation 5.8

$$
\begin{aligned}
\frac{\left|\psi^{\prime}-\phi\right|}{\left|\psi^{\prime}\right|+|\phi|}= & \frac{\frac{\lambda}{p^{2}|\phi|^{2}+2 m p \lambda+r \lambda^{2}} \sqrt{|\phi|^{2}(m p+r \lambda)^{2}+\left(p|\phi|^{2}+m \lambda\right)^{2} r-2(m p+r \lambda)\left(p|\phi|^{2}+m \lambda\right) m}}{\frac{p|\phi|^{2}+m \lambda}{p^{2}|\phi|^{2}+2 m p \lambda+r \lambda^{2}} \sqrt{p^{2}|\phi|^{2}+r \lambda^{2}+2 m p \lambda}+|\phi|} \\
& =\frac{\lambda \sqrt{|\phi|^{2}(m p+r \lambda)^{2}+\left(p|\phi|^{2}+m \lambda\right)^{2} r-2(m p+r \lambda)\left(p|\phi|^{2}+m \lambda\right) m}}{\left(p|\phi|^{2}+m \lambda\right) \sqrt{p^{2}|\phi|^{2}+r \lambda^{2}+2 m p \lambda}+\left(p^{2}|\phi|^{2}+2 m p \lambda+r \lambda^{2}\right)|\phi|}
\end{aligned}
$$

However, this equation depends on $|\phi|, m$, and $r$ which would not be known for real applications. To account for these variables, we will introduce a new parameter, $c$ :

$$
c=|\phi| \frac{\sqrt{r}}{m}
$$

While $c$ may also be unknown, we will be able to take a maximum of the resulting error function to get a bound. Using the bounds of L2-norm in terms of the L1-norm and noting that the L1-norm is equal to $m$, we obtain the identity

$$
1 \leq c \leq \sqrt{r}
$$

By substituting, $|\phi|=c \frac{m}{\sqrt{r}}$ and a bit of algebra, we can transform Equation A.10 into a final 
form:

$$
\begin{aligned}
\frac{\left|\psi^{\prime}-\phi\right|}{\left|\psi^{\prime}\right|+|\phi|}= & \frac{\lambda \sqrt{|\phi|^{2}(m p+r \lambda)^{2}+\left(p|\phi|^{2}+m \lambda\right)^{2} r-2(m p+r \lambda)\left(p|\phi|^{2}+m \lambda\right) m}}{\left(p|\phi|^{2}+m \lambda\right) \sqrt{p^{2}|\phi|^{2}+r \lambda^{2}+2 m p \lambda}+\left(p^{2}|\phi|^{2}+2 m p \lambda+r \lambda^{2}\right)|\phi|} \\
= & \frac{\lambda \sqrt{c^{2} \frac{m^{2}}{r}(m p+r \lambda)^{2}+\left(p c^{2} \frac{m^{2}}{r}+m \lambda\right)^{2} r-2(m p+r \lambda)\left(p c^{2} \frac{m^{2}}{r}+m \lambda\right) m}}{\left(p c^{2} \frac{m^{2}}{r}+m \lambda\right) \sqrt{p^{2} c^{2} \frac{m^{2}}{r}+r \lambda^{2}+2 m p \lambda}+\left(p^{2} c^{2} \frac{m^{2}}{r}+2 m p \lambda+r \lambda^{2}\right) c \frac{m}{\sqrt{r}}} \\
= & \frac{\frac{1}{r \sqrt{r}} \lambda \sqrt{c^{2} \frac{m^{2}}{r}(m p+r \lambda)^{2}+\left(p c^{2} \frac{m^{2}}{r}+m \lambda\right)^{2} r-2(m p+r \lambda)\left(p c^{2} \frac{m^{2}}{r}+m \lambda\right) m}}{r \sqrt{r}\left(\left(p c^{2} \frac{m^{2}}{r}+m \lambda\right) \sqrt{p^{2} c^{2} \frac{m^{2}}{r}+r \lambda^{2}+2 m p \lambda}+\left(p^{2} c^{2} \frac{m^{2}}{r}+2 m p \lambda+r \lambda^{2}\right) c \frac{m}{\sqrt{r}}\right)} \\
= & \frac{\lambda \sqrt{c^{2} \frac{m^{2}}{r^{2}}\left(\frac{m}{r} p+\lambda\right)^{2}+\left(p c^{2} \frac{m^{2}}{r^{2}}+\frac{m}{r} \lambda\right)^{2}-2\left(\frac{m}{r} p+\lambda\right)\left(p c^{2} \frac{m^{2}}{r^{2}}+\frac{m}{r} \lambda\right) \frac{m}{r}}}{\left(p c^{2} \frac{m^{2}}{r^{2}}+\frac{m}{r} \lambda\right) \sqrt{p^{2} c^{2} \frac{m^{2}}{r^{2}}+\lambda^{2}+2 \frac{m}{r} p \lambda+\left(p^{2} c^{2} \frac{m^{2}}{r^{2}}+2 \frac{m}{r} p \lambda+\lambda^{2}\right) c \frac{m}{r}}} \\
= & \frac{\lambda \sqrt{c^{2} h^{2}(h p+\lambda)^{2}+\left(p c^{2} h^{2}+h \lambda\right)^{2}-2(h p+\lambda)\left(p c^{2} h^{2}+h \lambda\right) h}}{\left(p c^{2} h^{2}+h \lambda\right) \sqrt{p^{2} c^{2} h^{2}+\lambda^{2}+2 h p \lambda}+\left(p^{2} c^{2} h^{2}+2 h p \lambda+\lambda^{2}\right) c h} \\
= & \frac{\frac{1}{h^{2}} \lambda \sqrt{c^{2} h^{2}(h p+\lambda)^{2}+\left(p c^{2} h^{2}+h \lambda\right)^{2}-2(h p+\lambda)\left(p c^{2} h^{2}+h \lambda\right) h}}{\frac{1}{h^{2}}\left(\left(p c^{2} h^{2}+h \lambda\right) \sqrt{p^{2} c^{2} h^{2}+\lambda^{2}+2 h p \lambda}+\left(p^{2} c^{2} h^{2}+2 h p \lambda+\lambda^{2}\right) c h\right)} \\
= & \frac{\lambda}{h} \frac{\sqrt{c^{2}\left(p+\frac{\lambda}{h}\right)^{2}+\left(p c^{2}+\frac{\lambda}{h}\right)^{2}-2\left(p+\frac{\lambda}{h}\right)\left(p c^{2}+\frac{\lambda}{h}\right)}}{\left(p c^{2}+\frac{\lambda}{h}\right) \sqrt{p^{2} c^{2}+\left(\frac{\lambda}{h}\right)^{2}+2 p \frac{\lambda}{h}+\left(p^{2} c^{2}+2 p \frac{\lambda}{h}+\left(\frac{\lambda}{h}\right)^{2}\right) c}} \\
& \\
& \\
&
\end{aligned}
$$

Note that this formula is a function of $\frac{\lambda}{h}, p$, and $c$. By expanding this function as a Taylor series, we find

$$
\frac{\left|\psi^{\prime}-\phi\right|}{\left|\psi^{\prime}\right|+|\phi|}=\frac{\sqrt{c^{2}-1} \lambda}{2 c^{2} h p}-\frac{\sqrt{c^{2}-1} \lambda^{2}}{2 c^{4} h^{2} p^{2}}+\ldots
$$

Noting that $\frac{\sqrt{c^{2}-1} \lambda^{2}}{2 c^{4} h^{2} p^{2}}$ is always positive and applying Taylor's theorem, we end up with the inequality

$$
\frac{\left|\psi^{\prime}-\phi\right|}{\left|\psi^{\prime}\right|+|\phi|} \leq \frac{\sqrt{c^{2}-1} \lambda}{2 c^{2} h p} \leq \frac{1}{4} \frac{\lambda}{h p}
$$

Where the last step comes from the inequality

$$
\frac{\sqrt{c^{2}-1}}{c^{2}} \leq \frac{1}{2}
$$




\section{Appendix B}

\section{Object Detection after Derain}

Sample of the results of the experiments described in Chapter 4 .

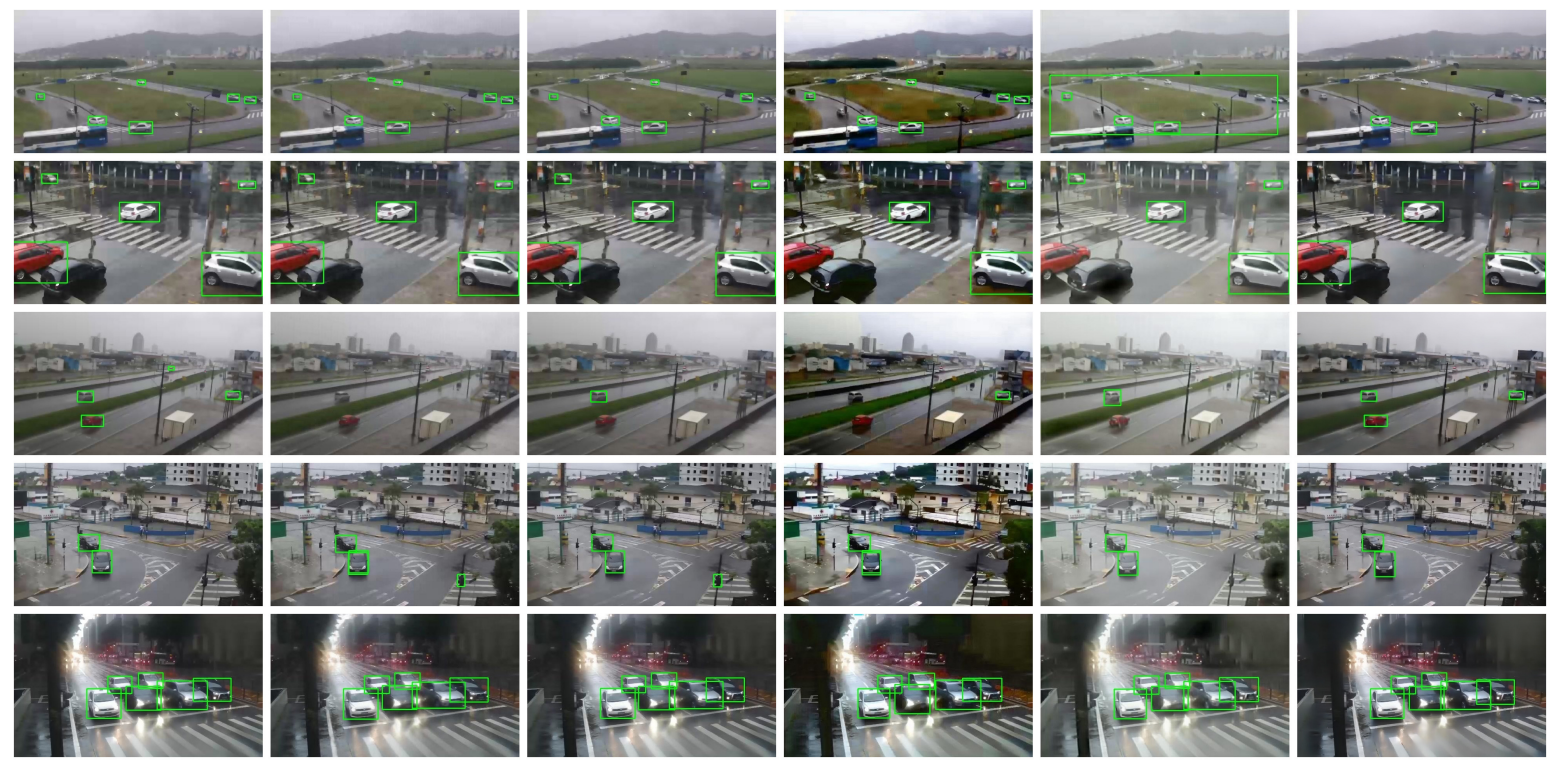

Figure B.1: Sample of the detection on rainy images. 
APPENDIX B 


\section{Bibliography}

[1] Ian F Akyildiz, Tommaso Melodia, and Kaushik R Chowdhury. A survey on wireless multimedia sensor networks. Computer Networks, 51(4):921-960, 2007. 22

[2] Ian F Akyildiz, Weilian Su, Yogesh Sankarasubramaniam, and Erdal Cayirci. A survey on sensor networks. IEEE Communications Magazine, 40(8):102-114, 2002. 22

[3] Amazon Web Services. Mechanical Turk, 2013. 5, 9

[4] Daniele Angiati, Gianluca Gera, Stefano Piva, and Carlo S Regazzoni. A novel method for graffiti detection using change detection algorithm. In International Conference on Advanced Video and Signal-based Surveillance, pages 242-246. IEEE, 2005. 38

[5] Pablo Arbeláez, Bharath Hariharan, Chunhui Gu, Saurabh Gupta, Lubomir Bourdev, and Jitendra Malik. Semantic segmentation using regions and parts. In Conference on Computer Vision and Pattern Recognition, pages 3378-3385. IEEE, 2012. 38

[6] Blue Archer. Graffiti Tracking system. http://www.graffititrackingsystem.com/, 2005. [Last accessed April-2018]. 37

[7] Sean M Arietta, Alexei A Efros, Ravi Ramamoorthi, and Maneesh Agrawala. City forensics: Using visual elements to predict non-visual city attributes. IEEE Transactions on Visualization and Computer Graphics, 20(12):2624-2633, 2014. 22

[8] Automated Regional Justice Information System (ARJIS). Graffiti tracker. http:// graffititracker.net/, 2006. [Last accessed April-2018]. 37

[9] Vijay Badrinarayanan, Alex Kendall, and Roberto Cipolla. Segnet: A deep convolutional encoder-decoder architecture for image segmentation. arXiv preprint arXiv:1511.00561, 2015. 38

[10] Michael Baron. Probability and statistics for computer scientists. pages 256-257. CRC Press, 2 edition, 2014. 10

[11] Stefano Basagni, Alessio Carosi, and Chiara Petrioli. Controlled vs. uncontrolled mobility in wireless sensor networks: Some performance insights. In Vehicular Technology Conference, pages 269-273, Maryland, USA, 2007. IEEE. 22

[12] Stefan Behnel, Robert Bradshaw, Craig Citro, Lisandro Dalcin, Dag Sverre Seljebotn, and Kurt Smith. Cython: The best of both worlds. Computing in Science Engineering, 13(2):31 $-39,2011.33$

[13] Jon Louis Bentley. Multidimensional binary search trees used for associative searching. Communications of the ACM, 18(9):509-517, 1975. 30

[14] Christopher JC Burges. A tutorial on support vector machines for pattern recognition. Data mining and knowledge discovery, 2(2):121-167, 1998. 6, 22

[15] Camerite. http://www.camerite.com/. [Last accessed March-2017]. 11 
[16] Tracy Camp, Jeff Boleng, and Vanessa Davies. A survey of mobility models for ad hoc network research. Wireless Communications and Mobile Computing, 2(5):483-502, 2002. 23, 36

[17] Olivier Chapelle, Bernhard Scholkopf, and Alexander Zien. Semi-supervised learning. Transactions on Neural Networks, 20(3):542-542, 2009. 5, 9

[18] CL Philip Chen and Chun-Yang Zhang. Data-intensive applications, challenges, techniques and technologies: A survey on big data. Information Sciences, 275:314-347, 2014. 1

[19] Yi-Lei Chen and Chiou-Ting Hsu. A generalized low-rank appearance model for spatiotemporally correlated rain streaks. In International Conference on Computer Vision, pages 1968-1975, 2013. 16

[20] Kelly J Clifton, Andrea D Livi Smith, and Daniel Rodriguez. The development and testing of an audit for the pedestrian environment. Landscape and Urban Planning, 80(1-2):95-110, 2007. 1

[21] Sunny Consolvo, David W McDonald, Tammy Toscos, Mike Y Chen, Jon Froehlich, Beverly Harrison, Predrag Klasnja, Anthony LaMarca, Louis LeGrand, Ryan Libby, et al. Activity sensing in the wild: a field trial of ubifit garden. In Conference on Human Factors in Computing Systems, pages 1797-1806, Florence,Italy, 2008. ACM. 22

[22] Marius Cordts, Mohamed Omran, Sebastian Ramos, Timo Rehfeld, Markus Enzweiler, Rodrigo Benenson, Uwe Franke, Stefan Roth, and Bernt Schiele. The cityscapes dataset for semantic urban scene understanding. In Conference on Computer Vision and Pattern Recognition, pages 3213-3223, Nevada, USA, 2016. IEEE. 22, 23, 39

[23] CrowdFlower Inc. https://www.crowdflower.com, Last accessed March 2017. 9

[24] Jifeng Dai, Kaiming He, Yi Li, Shaoqing Ren, and Jian Sun. Instance-sensitive fully convolutional networks. In European Conference on Computer Vision, pages 534-549. Springer, 2016. 38

[25] Jifeng Dai, Yi Li, Kaiming He, and Jian Sun. R-FCN: Object detection via region-based fully convolutional networks. arXiv preprint arXiv:1605.06409, 2016. x, 22, 25, 28, 29

[26] Navneet Dalal and Bill Triggs. Histograms of oriented gradients for human detection. In Conference on Computer Vision and Pattern Recognition, volume 1, pages 886-893, California, USA, 2005. IEEE. 6, 22

[27] Vanessa Ann Davies et al. Evaluating mobility models within an ad hoc network. Master's thesis, Citeseer, 2000. 23

[28] Luigi Di Stefano, Federico Tombari, Alessandro Lanza, Stefano Mattoccia, and Stefano Monti. Graffiti detection using two views. In International Workshop on Visual Surveillance, 2008. 38

[29] Carl Doersch, Saurabh Singh, Abhinav Gupta, Josef Sivic, and Alexei Efros. What makes paris look like paris? ACM Transactions on Graphics, 31(4), 2012. 38

[30] Mark Everingham, Luc Van Gool, Christopher KI Williams, John Winn, and Andrew Zisserman. The pascal visual object classes challenge. International Journal of Computer Vision, 88(2):303-338, 2010. x, 7, 17, 23, 25, 28, 29, 39

[31] Pedro F Felzenszwalb, Ross B Girshick, David McAllester, and Deva Ramanan. Object detection with discriminatively trained part-based models. IEEE Transactions on Pattern Analysis and Machine Intelligence, 32(9):1627-1645, 2010. 6, 9, 10, 12, 13 
[32] Nivan Ferreira, Jorge Poco, Huy T Vo, Juliana Freire, and Cláudio T Silva. Visual exploration of big spatio-temporal urban data: A study of new york city taxi trips. IEEE Transactions on Visualization and Computer Graphics, 19(12):2149-2158, 2013. 1

[33] Xueyang Fu, Jiabin Huang, Delu Zeng, Yue Huang, Xinghao Ding, and John Paisley. Removing rain from single images via a deep detail network. In Conference on Computer Vision and Pattern Recognition, 2017. 16, 17

[34] Silvia Gandy, Benjamin Recht, and Isao Yamada. Tensor completion and low-n-rank tensor recovery via convex optimization. Inverse Problems, 27(2):025010, 2011. 36

[35] Chenqiang Gao, Pei Li, Yajun Zhang, Jiang Liu, and Lan Wang. People counting based on head detection combining adaboost and $\mathrm{cnn}$ in crowded surveillance environment. Neurocomputing, 208:108-116, 2016. 22

[36] Andreas Geiger, Philip Lenz, Christoph Stiller, and Raquel Urtasun. Vision meets robotics: The kitti dataset. International Journal of Robotics Research (IJRR), 2013. 22, 23

[37] Ross Girshick, Jeff Donahue, Trevor Darrell, and Jitendra Malik. Region-based convolutional networks for accurate object detection and segmentation. IEEE Transactions on Pattern Analysis and Machine Intelligence, 38(1):142-158, 2016. 7

[38] Google. Google maps. https://maps.google.com/, 2005. [Last accessed April-2018]. x, xiii, $22,23,37,38,39,40,42$

[39] Peter E Hart, Nils J Nilsson, and Bertram Raphael. A formal basis for the heuristic determination of minimum cost paths. IEEE Transactions on Systems Science and Cybernetics, 4(2):100-107, 1968. 27

[40] Kaiming He, Georgia Gkioxari, Piotr Dollár, and Ross Girshick. Mask r-cnn. In International Conference on Computer Vision, pages 2980-2988. IEEE, 2017. x, 38, 39, 40

[41] Kaiming He, Xiangyu Zhang, Shaoqing Ren, and Jian Sun. Deep residual learning for image recognition. In Conference on Computer Vision and Pattern Recognition. IEEE, 2016. x, 16, $22,28,29,39$

[42] Dirk Helbing. A fluid dynamic model for the movement of pedestrians. arXiv preprint condmat/9805213, 1998. 36

[43] Dirk Helbing. Traffic and related self-driven many-particle systems. Reviews of modern physics, $73(4): 1067,2001.36$

[44] Chi-Fu Huang and Yu-Chee Tseng. The coverage problem in a wireless sensor network. Mobile Networks and Applications, 10(4):519-528, 2005. 22

[45] Human Development Report Office (HDRO). Human Development Report: Concept and Measurement of Human Development. Technical Report ISBN 0-19-506480-1, United Nations Development Programme, 1990. 41

[46] Quan Huynh-Thu and Mohammed Ghanbari. Scope of validity of psnr in image/video quality assessment. Electronics letters, 44(13):800-801, 2008. 15

[47] Tomoharu Iwata, Hitoshi Shimizu, Futoshi Naya, and Naonori Ueda. Estimating people flow from spatiotemporal population data via collective graphical mixture models. Transactions on Spatial Algorithms and Systems, 3(1):2, 2017. 36

[48] Gareth James, Daniela Witten, Trevor Hastie, and Robert Tibshirani. An introduction to statistical learning, volume 112. Springer, 2013. 9 
[49] David B Johnson and David A Maltz. Dynamic source routing in ad hoc wireless networks. Mobile computing, 353(1):153-181, 1996. 23, 27

[50] Zdenek Kalal, Krystian Mikolajczyk, and Jiri Matas. Tracking-learning-detection. IEEE Transactions on Pattern Analysis and Machine Intelligence, 34(7):1409-1422, 2012. 10

[51] Sophia Karagiorgou, Dieter Pfoser, and Dimitrios Skoutas. A layered approach for more robust generation of road network maps from vehicle tracking data. Transactions on Spatial Algorithms and Systems, 3(1):3, 2017. 22

[52] Leonard Kleinrock. Queueing systems, volume 2: Computer applications, volume 66. wiley New York, 1976. 47

[53] Donald Ervin Knuth. The art of computer programming, volume 3. Pearson Education, 1997. 23

[54] Alex Krizhevsky et al. Learning multiple layers of features from tiny images. Technical report, Citeseer, 2009. 23

[55] Alex Krizhevsky, Ilya Sutskever, and Geoffrey E Hinton. Imagenet classification with deep convolutional neural networks. In Advances in neural information processing systems, pages 1097-1105, Nevada, USA, 2012. 7, 22

[56] Christoph H Lampert, Matthew B Blaschko, and Thomas Hofmann. Efficient subwindow search: A branch and bound framework for object localization. IEEE Transactions on Pattern Analysis and Machine Intelligence, 31(12):2129, 2009. 7

[57] Nicholas D Lane, Emiliano Miluzzo, Hong Lu, Daniel Peebles, Tanzeem Choudhury, and Andrew T Campbell. A survey of mobile phone sensing. IEEE Communications Magazine, 48(9):140-150, 2010. 22

[58] Averill M Law, W David Kelton, and W David Kelton. Simulation modeling and analysis, volume 3. McGraw-Hill New York, Arizona, USA, 2007. 27

[59] Yann LeCun, Yoshua Bengio, and Geoffrey Hinton. Deep learning. Nature, 521(7553):436, 2015. 6

[60] Uichin Lee, Eugenio Magistretti, Mario Gerla, Paolo Bellavista, and Antonio Corradi. Dissemination and harvesting of urban data using vehicular sensing platforms. IEEE Transactions on Vehicular Technology, 58(2):882-901, 2009. 23

[61] Victor Lesser, Charles L Ortiz Jr, and Milind Tambe. Distributed sensor networks: A multiagent perspective, volume 9. Springer Science \& Business Media, 2012. 23

[62] Boyi Li, Xiulian Peng, Zhangyang Wang, Jizheng Xu, and Dan Feng. Aod-net: All-in-one dehazing network. In International Conference on Computer Vision, 2017. 11

[63] Li Li, Yuebiao Li, and Zhiheng Li. Efficient missing data imputing for traffic flow by considering temporal and spatial dependence. Transportation research part C: emerging technologies, 34:108-120, 2013. 36

[64] Siyuan Li, Iago Breno Araujo, Wenqi Ren, Zhangyang Wang, Eric K. Tokuda, Roberto Hirata Junior, Roberto Cesar-Junior, Jiawan Zhang, Xiaojie Guo, and Xiaochun Cao. Single image deraining: A comprehensive benchmark analysis. In Conference on Computer Vision and Pattern Recognition, 2019. 15

[65] Weizi Li, David Wolinski, and Ming C Lin. City-scale traffic animation using statistical learning and metamodel-based optimization. ACM Transactions on Graphics, 36(6):200, 2017. 22, 36 
[66] Xiaojiang Li, Chuanrong Zhang, Weidong Li, Robert Ricard, Qingyan Meng, and Weixing Zhang. Assessing street-level urban greenery using google street view and a modified green view index. Urban Forestry $\mathscr{G}$ Urban Greening, 14(3):675-685, 2015. 38

[67] Yu Li, Robby T Tan, Xiaojie Guo, Jiangbo Lu, and Michael S Brown. Rain streak removal using layer priors. In Conference on Computer Vision and Pattern Recognition, pages 27362744, 2016. 16

[68] Ben Liang and Zygmunt J Haas. Predictive distance-based mobility management for pcs networks. In International Conference on Computer Communications, volume 3, pages 13771384, New York, USA, 1999. IEEE. 23

[69] Tsung-Yi Lin, Priyal Goyal, Ross Girshick, Kaiming He, and Piotr Dollár. Focal loss for dense object detection. IEEE Transactions on Pattern Analysis and Machine Intelligence, 2018. 7, 17,42

[70] Tsung-Yi Lin, Michael Maire, Serge Belongie, James Hays, Pietro Perona, Deva Ramanan, Piotr Dollár, and C Lawrence Zitnick. Microsoft coco: Common objects in context. In European Conference on Computer Vision, pages 740-755. Springer, 2014. 23, 39

[71] Lixiong Liu, Bao Liu, Hua Huang, and Alan Conrad Bovik. No-reference image quality assessment based on spatial and spectral entropies. Signal Processing: Image Communication, $29(8): 856-863,2014.16,17$

[72] Wei Liu, Dragomir Anguelov, Dumitru Erhan, Christian Szegedy, Scott Reed, Cheng-Yang $\mathrm{Fu}$, and Alexander C Berg. Ssd: Single shot multibox detector. In European Conference on Computer Vision, pages 21-37, 2016. 7, 17

[73] 594 Graffiti LLC. Tracking and Automated Graffiti Reporting System (TAGRS). http: //www.594graffiti.com, 2009. [Last accessed April-2018]. 37

[74] Jonathan Long, Evan Shelhamer, and Trevor Darrell. Fully convolutional networks for semantic segmentation. In Conference on Computer Vision and Pattern Recognition, pages 3431-3440, 2015. 38

[75] David G Lowe. Object recognition from local scale-invariant features. In Computer vision, 1999. The proceedings of the seventh IEEE international conference on, volume 2, pages 1150 1157. Ieee, 1999. 6

[76] Yu Luo, Yong $\mathrm{Xu}$, and Hui Ji. Removing rain from a single image via discriminative sparse coding. In International Conference on Computer Vision, 2015. 16

[77] Will Maddern, Geoffrey Pascoe, Chris Linegar, and Paul Newman. 1 year, 1000 km: The oxford robotcar dataset. The International Journal of Robotics Research, 36(1):3-15, 2017. 22,23

[78] Makoto Matsumoto and Takuji Nishimura. Mersenne twister: a 623-dimensionally equidistributed uniform pseudo-random number generator. Transactions on Modeling and Computer Simulation, 8(1):3-30, 1998. 23, 33

[79] Cameron McAuliffe. Graffiti or street art? negotiating the moral geographies of the creative city. Journal of urban affairs, 34(2):189-206, 2012. 37

[80] Fabio Miranda, Harish Doraiswamy, Marcos Lage, Luc Wilson, Mondrian Hsieh, and Claudio T Silva. Shadow accrual maps: Efficient accumulation of city-scale shadows over time. IEEE Transactions on Visualization and Computer Graphics, 2018. 36 
[81] Ishan Misra, Abhinav Shrivastava, and Martial Hebert. Watch and learn: Semi-supervised learning for object detectors from video. In Conference on Computer Vision and Pattern Recognition, pages 3593-3602, 2015. 9

[82] Anish Mittal, Rajiv Soundararajan, and Alan C Bovik. Making a" completely blind" image quality analyzer. IEEE Signal Processing Letters, 20(3):209-212, 2013. 16, 17

[83] Jorge MC Mondego, Ramon O Vidal, Marcelo F Carazzolle, Eric K Tokuda, Lucas P Parizzi, Gustavo GL Costa, Luiz FP Pereira, Alan C Andrade, Carlos A Colombo, Luiz GE Vieira, et al. An est-based analysis identifies new genes and reveals distinctive gene expression features of coffea arabica and coffea canephora. BMC plant biology, 11(1):30, 2011. 9

[84] New York Times. Inside China's Dystopian Dreams: A.I., Shame and Lots of Cameras. https: //www.nytimes.com/2018/07/08/business/china-surveillance-technology.html, 2018. [Last accessed August-2018]. 2

[85] Muaz A Niazi and Amir Hussain. A novel agent-based simulation framework for sensing in complex adaptive environments. IEEE Sensors Journal, 11(2):404-412, 2011. 23

[86] NYC open data. (https://opendata.cityofnewyork.us/), Last accessed March 2017. 36

[87] Sangmin Oh, Anthony Hoogs, Amitha Perera, Naresh Cuntoor, Chia-Chih Chen, Jong Taek Lee, Saurajit Mukherjee, JK Aggarwal, Hyungtae Lee, Larry Davis, et al. A large-scale benchmark dataset for event recognition in surveillance video. In Conference on Computer Vision and Pattern Recognition, pages 3153-3160, Colorado, USA, 2011. IEEE. 22, 23

[88] OpenStreetMap community. OpenStreetMap. https://www.openstreetmap.org, 2004. [Last accessed August-2018]. x, 30

[89] Yair E Orgler. A credit scoring model for commercial loans. Journal of money, Credit and Banking, 2(4):435-445, 1970. 1

[90] Mohd Fauzi Othman and Khairunnisa Shazali. Wireless sensor network applications: A study in environment monitoring system. Procedia Engineering, 41:1204-1210, 2012. 22

[91] Stephen K. Park and Keith W. Miller. Random number generators: good ones are hard to find. Communications of the ACM, 31(10):1192-1201, 1988. 23

[92] Photosynth. (https://blogs.msdn.microsoft.com/photosynth/2017/02/06/microsoftphotosynth-has-been-shut-down/), Last accessed March 2017. 36

[93] Pedro O Pinheiro, Ronan Collobert, and Piotr Dollár. Learning to segment object candidates. In Advances in Neural Information Processing Systems, pages 1990-1998, 2015. 38

[94] United Nations Human Settlements Programme. The challenge of slums global report on human settlements 2003. Technical report, UN-Habitat, 2003. 1

[95] Rui Qian, Robby T Tan, Wenhan Yang, Jiajun Su, and Jiaying Liu. Attentive generative adversarial network for raindrop removal from a single image. In Conference on Computer Vision and Pattern Recognition, 2018. 16, 17

[96] Rajib Kumar Rana, Chun Tung Chou, Salil S Kanhere, Nirupama Bulusu, and Wen Hu. Earphone: an end-to-end participatory urban noise mapping system. In International Conference on Information Processing in Sensor Networks, pages 105-116. ACM, 2010. 22

[97] Jonathan Reades, Francesco Calabrese, Andres Sevtsuk, and Carlo Ratti. Cellular census: Explorations in urban data collection. IEEE Pervasive computing, 6(3):30-38, 2007. 22 
[98] Joseph Redmon, Santosh Divvala, Ross Girshick, and Ali Farhadi. You only look once: Unified, real-time object detection. In Conference on Computer Vision and Pattern Recognition, pages 779-788, 2016. 7

[99] Joseph Redmon and Ali Farhadi. Yolov3: An incremental improvement. arXiv preprint arXiv:1804.0276\%, 2018. 17

[100] Shaoqing Ren, Kaiming He, Ross Girshick, and Jian Sun. Faster r-cnn: Towards real-time object detection with region proposal networks. In Advances in neural information processing systems, pages $91-99,2015.7,9,17,22,38,39$

[101] Olaf Ronneberger, Philipp Fischer, and Thomas Brox. U-net: Convolutional networks for biomedical image segmentation. In International Conference on Medical image computing and computer-assisted intervention, pages 234-241. Springer, 2015. 38

[102] Chuck Rosenberg, Martial Hebert, and Henry Schneiderman. Semi-supervised self-training of object detection models. In Workshops on Application of Computer Vision - Volume 01. IEEE, 2005. 9

[103] Andrew G Rundle, Michael DM Bader, Catherine A Richards, Kathryn M Neckerman, and Julien O Teitler. Using google street view to audit neighborhood environments. American Journal of Preventive Medicine, 40(1):94-100, 2011. 38

[104] Olga Russakovsky, Jia Deng, Hao Su, Jonathan Krause, Sanjeev Satheesh, Sean Ma, Zhiheng Huang, Andrej Karpathy, Aditya Khosla, Michael Bernstein, et al. Imagenet large scale visual recognition challenge. International Journal of Computer Vision, 115(3):211-252, 2015. 7, 9, 10,23

[105] Michele A Saad, Alan C Bovik, and Christophe Charrier. Blind image quality assessment: A natural scene statistics approach in the det domain. IEEE Transactions on Image Processing, 21(8):3339-3352, 2012. 16, 17

[106] Muhammad Saqib, Sultan Daud Khan, Nabin Sharma, and Michael Blumenstein. Person head detection in multiple scales using deep convolutional neural networks. In International Joint Conference on Neural Networks, pages 1-7. IEEE, 2018. 22

[107] Linda See, Alexis Comber, Carl Salk, Steffen Fritz, Marijn van der Velde, Christoph Perger, Christian Schill, Ian McCallum, Florian Kraxner, and Michael Obersteiner. Comparing the quality of crowdsourced data contributed by expert and non-experts. PloS one, 8(7):e69958, 2013. 9

[108] Xiang Sheng, Jian Tang, and Weiyi Zhang. Energy-efficient collaborative sensing with mobile phones. In International Conference on Computer Communications, pages 1916-1924, Florida, USA, 2012. IEEE. 22

[109] Wenhuan Shi, Shuhan Shen, and Yuncai Liu. Automatic generation of road network map from massive gps, vehicle trajectories. In Intelligent Transportation Systems, 2009. ITSC'09. 12th International IEEE Conference on, pages 1-6, Missouri, USA, 2009. IEEE. 22

[110] Ashish Shrivastava, Tomas Pfister, Oncel Tuzel, Josh Susskind, Wenda Wang, and Russ Webb. Learning from simulated and unsupervised images through adversarial training. arXiv preprint arXiv:1612.07828, 2016. 13

[111] Karen Simonyan and Andrew Zisserman. Very deep convolutional networks for large-scale image recognition. arXiv preprint arXiv:1409.1556, 2014. 12

[112] Alexander Sorokin and David Forsyth. Utility data annotation with amazon mechanical turk. In Computer Vision and Pattern Recognition Workshops. IEEE, 2008. 9 
[113] Stephen V Stehman. Basic probability sampling designs for thematic map accuracy assessment. International Journal of remote sensing, 20(12):2423-2441, 1999. 38

[114] Thomas Stockhammer. Dynamic adaptive streaming over http-: standards and design principles. In Conference on Multimedia systems, pages 133-144. ACM, 2011. 11

[115] Christian Szegedy, Wei Liu, Yangqing Jia, Pierre Sermanet, Scott Reed, Dragomir Anguelov, Dumitru Erhan, Vincent Vanhoucke, Andrew Rabinovich, et al. Going deeper with convolutions. In Conference on Computer Vision and Pattern Recognition, Massachusetts, USA, 2015. 22

[116] Richard Szeliski. Computer vision: algorithms and applications. Springer Science \& Business Media, 2010. 2, 5, 6, 9

[117] São Paulo City Hall. Atlas do Trabalho de Desenvolvimento da Cidade de São Paulo 2012. http://atlasmunicipal.prefeitura.sp.gov.br/, 2012. [Last accessed Nov-2017]. xi, 41

[118] Jing Tian, Jorg Hahner, Christian Becker, Illya Stepanov, and Kurt Rothermel. Graph-based mobility model for mobile ad hoc network simulation. In Simulation Symposium, pages 337344, California, USA, 2002. IEEE. 27

[119] Ben L Titzer, Daniel K Lee, and Jens Palsberg. Avrora: Scalable sensor network simulation with precise timing. In Information Processing in Sensor Networks, 2005. IPSN 2005. Fourth International Symposium on, pages 477-482, Tennessee, USA, 2005. IEEE. 23

[120] Eric K. Tokuda, Roberto M. Cesar-Jr., and Claudio T. Silva. Quantifying the presence of graffiti in urban environments. In International Conference on Big Data and Smart Computing, Japan, 2019. IEEE. 36, 37

[121] Eric K. Tokuda, Gabriel B. A. Ferreira, Claudio Silva, and Roberto M. Cesar-Jr. A novel semi-supervised detection approach with weak annotation. In Southwest Symposium on Image Analysis and Interpretation, Nevada, USA, 2018. IEEE. 9

[122] Eric K Tokuda, Yitzchak Lockerman, Gabriel BA Ferreira, Ethan Sorrelgreen, David Boyle, Roberto M Cesar-Jr, and Claudio T Silva. A new approach for pedestrian density estimation using moving sensors and computer vision. ACM Transactions on Spatial Algorithms and Systems (TSAS), 6(4):1-20, 2020. 21

[123] Eric K. Tokuda, Helio Pedrini, and Anderson Rocha. Computer generated images vs. digital photographs: A synergetic feature and classifier combination approach. Journal of Visual Communication and Image Representation, 24(8):1276-1292, 2013. 13

[124] Federico Tombari, Luigi Di Stefano, Stefano Mattoccia, and Andrea Zanetti. Graffiti detection using a time-of-flight camera. In International Conference on Advanced Concepts for Intelligent Vision Systems, pages 645-654. Springer, 2008. 38

[125] Wei Tong, Jung-Eun Lee, Rong Jin, and Anil K Jain. Gang and moniker identification by graffiti matching. In International Workshop on Multimedia in Forensics and Intelligence, pages 1-6. ACM, 2011. 38

[126] Akihiko Torii, Michal Havlena, and Tomáš Pajdla. From google street view to 3d city models. In International Conference on Computer vision workshops, pages 2188-2195. IEEE, 2009. 38

[127] Abhishek Kumar Tripathi and Sudipta Mukhopadhyay. Removal of rain from videos: a review. Signal, Image and Video Processing, 8(8):1421-1430, 2014. 15

[128] Jasper RR Uijlings, Koen EA Van De Sande, Theo Gevers, and Arnold WM Smeulders. Selective search for object recognition. International journal of computer vision, 104, 2013. 7 
[129] United Nations. World Urbanization Prospects: The 2018 Revision. Technical report, Population Division of the United Nations Department of Economic and Social Affairs (UN DESA), 2018. 1

[130] United States Environment Protection Agency Air Data USEEPAAD. https://www3.epa. gov/airdata/ad_data_daily.html, 2016. [Last accessed April-2018]. 22

[131] VandalTrak Ltd. VandalTrack. https://www.vandaltrak.com.au/, 2008. [Last accessed April2018]. 37

[132] Carlos A Vanegas, Daniel G Aliaga, and Bedrich Benes. Automatic extraction of manhattanworld building masses from 3d laser range scans. IEEE Transactions on Visualization and Computer Graphics, 18(10):1627-1637, 2012. 22

[133] Roberto Vezzani and Rita Cucchiara. Video surveillance online repository (visor): an integrated framework. Multimedia Tools and Applications, 50(2):359-380, 2010. 22, 23

[134] Meritxell Vinyals, Juan A Rodriguez-Aguilar, and Jesus Cerquides. A survey on sensor networks from a multiagent perspective. The Computer Journal, 54(3):455-470, 2011. 23

[135] Guiling Wang, Guohong Cao, and Tom LaPorta. A bidding protocol for deploying mobile sensors. In International Conference on Network Protocols, pages 315-324, Georgia, USA, 2003. IEEE. 22

[136] Zhou Wang, Alan C Bovik, Hamid R Sheikh, Eero P Simoncelli, et al. Image quality assessment: from error visibility to structural similarity. IEEE Transactions on Image Processing, 13(4):600-612, 2004. 15

[137] William H Whyte. City: Rediscovering the center. University of Pennsylvania Press, Pennsylvania, USA, 2012. 22

[138] Wikimedia. Wikimedia commons, the free media repository. https://commons.wikimedia. org/, 2005. [Last accessed April-2018]. ix, 2, 15

[139] Chunlei Yang, Pak Chung Wong, William Ribarsky, and Jianping Fan. Efficient graffiti image retrieval. In International Conference on Multimedia Retrieval, page 36. ACM, 2012. 38

[140] Danny B Yang, Leonidas J Guibas, et al. Counting people in crowds with a real-time network of simple image sensors. In International Conference on Computer Vision Workshops, page 122, Nice, France, 2003. IEEE. 22

[141] Wenhan Yang, Robby T Tan, Jiashi Feng, Jiaying Liu, Zongming Guo, and Shuicheng Yan. Deep joint rain detection and removal from a single image. In Conference on Computer Vision and Pattern Recognition, 2017. 16, 17

[142] Alison Young. Street art, public city: Law, crime and the urban imagination. Routledge, 2013. 37

[143] Eyasu Zemene, Yonatan Tariku Tesfaye, Haroon Idrees, Andrea Prati, Marcello Pelillo, and Mubarak Shah. Large-scale image geo-localization using dominant sets. IEEE Transactions on Pattern Analysis and Machine Intelligence, 41(1):148-161, 2018. 22

[144] He Zhang and Vishal M Patel. Density-aware single image de-raining using a multi-stream dense network. In Conference on Computer Vision and Pattern Recognition, 2018. 16, 17

[145] He Zhang, Vishwanath Sindagi, and Vishal M Patel. Image de-raining using a conditional generative adversarial network. arXiv preprint arXiv:1701.05957, 2017. 16, 17 
[146] Xiaopeng Zhang, Hao Li, Yingyi Qi, Wee Kheng Leow, and Teck Khim Ng. Rain removal in video by combining temporal and chromatic properties. In International Conference on Multimedia and Expo, pages 461-464. IEEE, 2006. 11

[147] Yu Zheng, Licia Capra, Ouri Wolfson, and Hai Yang. Urban computing: concepts, methodologies, and applications. ACM Transactions on Intelligent Systems and Technology, 5(3):38, 2014. 22

[148] Bolei Zhou, Hang Zhao, Xavier Puig, Sanja Fidler, Adela Barriuso, and Antonio Torralba. Scene parsing through ade20k dataset. In Conference on Computer Vision and Pattern Recognition, 2017. 38

[149] Zhi-Hua Zhou. A brief introduction to weakly supervised learning. National Science Review, $5(1): 44-53,2017.5$ 